\title{
Option Prices with Uncertain Fundamentals Theory and Evidence on the Dynamics of Implied Volatilities*
}

\author{
Alexander David \\ Board of Governors of the \\ Federal Reserve System
}

\author{
Pietro Veronesi \\ Graduate School of Business \\ University of Chicago
}

This Version: August 1999

First Version: November 1998

Comments welcome

\footnotetext{
${ }^{*}$ We thank John Cochrane, George Constantinides, Gabrielle Demange, Darrell Duffie, Rick Green, Hayne Leland, Hong Yan and the seminar participants at Carnegie Mellon, Chicago, Federal Reserve Board, INSEAD, London Business School, McGill, Princeton, Stanford, Stockholm, Washington, the 9th Annual Derivatives Securities Conference held at Boston University, 1999 European Summer Symposium in Financial Markets at Gerzensee and the 1999 Rodos Economic Theory Conference for their comments and discussion. We also thank Gary Anderson for invaluable advice on Mathematica programming, Yubo Wang for his help with options data, and Jim Hamilton for sharing his Gauss code on regime-switching models. This paper represents the views of the authors and should not be interpreted as reflecting the views of the Board of Governors of the Federal Reserve System or its staff. Address (David): Mail Stop \# 90, Federal Reserve Board, Washington D.C. 20551. Phone: (202) 452-2333. Fax: (202) 452-5296. E. Mail: adavid@frb.gov. Address (Veronesi): Graduate School of Business, University of Chicago, Chicago, IL 60637. E. Mail: fpverone@gsbux1.uchicago.edu. Phone: (773) 702-6348. Fax: 773 702-0458. Veronesi gratefully acknowledges support from the Dimensional Fund Advisors Research Fund at the Graduate School of Business, University of Chicago.
} 


\begin{abstract}
In an incomplete information model, investors' uncertainty about the underlying drift rate of a firm's fundamentals affects option prices through $(i)$ endogenous and belief-dependent stochastic volatility, (ii) stochastic covariance between returns and volatility, and (iii) a market price of "belief risk." For the special case where the drift takes only two values, we provide an option pricing formula using Fourier Transforms. The model calibrated to 1960-1998 S\&P 500 real earnings growth shows that investors' uncertainty explains intertemporal variation in the slope and curvature of implied volatility curves as well as the conditional moments of the state-return density obtained from option data. The calibrated model generates hedging 'violations' of onefactor markov and deterministic volatility function models with roughly empirical frequencies.
\end{abstract}

JEL Classification: G12, G13

Key Words: Uncertainty, Changing Return-Volatility Correlation, Belief Risk, Put-Call Ratio, Butterfly Spread, Hedging Violations 


\section{Introduction}

The empirical financial literature has established a number of stylized facts about the intertemporal behavior of option prices that appear puzzling at first. For example, it has been observed that options' implied volatility curves are most often negatively sloped with a "smile" (or "smirk") and that they are highly unstable over time, with sudden changes in slope and shape. ${ }^{1}$ In fact, they sometimes become positively sloped or show a "frown" rather than a "smile." Figure 1 illustrates the range of observed implied volatility curves. Indeed, using middle-of-the month data on S\&P 500 index options between 1986 to 1996 , we find that the slope of the implied volatility curve computed at the point where the current stock price equals the strike price (i.e. for at-the-money options) was negative and/or had a positive first derivative ("smile") $88 \%$ and $78 \%$ of the time, respectively. However, the slope and the curvature flipped sign 15 and 35 times, respectively, out of the 122 monthly observations. In addition, it has been observed that the responses of option prices to changes in stock prices are inconsistent with single-factor markov and deterministic volatility function models. For example, Bakshi, Cao and Chen (1999) find that when the stock price increases, oftentimes the call prices decrease or they increase too much.

A number of empirical and theoretical papers have proposed models for stock prices to account for this behavior. For example, in their classic and influential paper Hull and White (1987) assume that stock return volatility varies stochastically over time and show that a negative (positive) correlation between returns and volatility tends to generate a negatively (positively) sloped implied volatility curve. Similarly, the possibility that stock prices jump at random times also may help explain some of the stylized facts mentioned above. (See Bakshi, Cao and Chen (1997) for a summary of these approaches.)

Although these models are useful for pricing purposes, they do not address the basic economic question of why changes in volatility and changes in the correlation between returns and volatility occur in the first place. This is because they start from a model in "reduced form": they assume a set of properties for the stochastic process of stock prices and derive pricing implications. Indeed, the need for an economic model explaining these phenomena has been realized in the

\footnotetext{
${ }^{1}$ The implied volatility of an option refers to the volatility level that has to be inserted into the Black and Scholes formula in order for it to match the price of a traded option using as inputs the current stock price, dividend yield, interest rate, and the strike price and maturity of that option contract. The implied volatility curve refers to the levels of implied volatilities across strike prices. Black and Scholes assumptions predict a constant implied volatility curve.
} 
literature: For example, discussing the results in Rubinstein (1985) about the sudden changes in the slope of the implied volatility curve, Hull and White (1987) write:

In order for [Rubinstein's (1985) results] to support this model it is necessary to posit that from one year [1976-77] to the next [1977-78], the correlation between stock prices and the associated volatility reversed sign. It is difficult to think of a convincing story why this event should occur. (Page 298, Hull and White 1987)

This paper constructs a simple dynamic asset pricing model to provide an economic justification for this and the other stylized facts about the intertemporal behavior of option prices that were mentioned above. Specifically, we assume that dividends of a firm (or of the economy as a whole) are generated by a standard lognormal process, whose drift rate is not observable by investors. The drift rate can be assumed constant or it may change over time according to a known stochastic process. Investors observe past realizations of dividends and possibly other signals and use these data to estimate the true drift rate of the dividend process.

Under these assumptions, we first solve for equilibrium stock prices and show that equilibrium stock returns display stochastic volatility, stochastic covariance between returns and volatility and a stochastic dividend yield. These properties of the stock return process are due to investors' changes over time of $(i)$ their estimate of the underlying drift rate of dividends and ( $i i)$ their confidence in this estimate. For example, we find that when uncertainty over the true drift rate of dividends is high, then return volatility is also high. ${ }^{2}$ Because investors' uncertainty changes randomly over time in response to news in dividend and other signals, return volatility also changes stochastically over time. Similarly, (i) and (ii) above also affect the covariance between returns and volatility. For example, suppose that at some point in time investors have a high confidence in a high drift rate of dividends. A series of negative innovations in dividends has two effects: the first is that the stock will move downward, generating a series of negative returns. The second effect is that, at the same time, investors' confidence in a high drift rate decreases, thereby increasing their uncertainty and hence the equilibrium return volatility. These effects together generate a negative correlation between returns and volatility. Similarly, if investors have high confidence in

\footnotetext{
${ }^{2}$ We make a slight but often used distinction between risk and uncertainty: we reserve the term uncertainty for situations where the risky process generating fundamentals in not fully known by agents. Whether this is the key distinction between risk and uncertainty made by Frank Knight is a subject of interpretation and debate; see Jones and Ostroy (1984) for a further discussion and references to the literature.
} 
a low estimate for the dividend drift rate and observe a series of high dividend realizations, we obtain a positive correlation between returns and volatility. In conclusion, the updating of investors' estimate of the dividend drift rate as well as their confidence in it over time make the correlation between return and volatility change stochastically over time. Since negative (positive) correlation between return and volatility tend to generate a negative (positive) slope in the implied volatility curve, this finding provides a simple explanation for the "puzzling" behavior of the implied volatility curve pointed out in the quote above.

This example also provides the intuition on why this model may generate very high or very low sensitivity of option prices to changes in stock prices. A negative innovation in dividends reduces the stock price and hence it has a direct negative effect on the call price. However, if the negative dividend innovation also increases the uncertainty and hence the volatility of stock returns, it will have an indirect positive effect on the call price. It could be the case that the indirect effect is stronger than the direct effect, thereby generating a negative covariance between stock price and call price changes; this violation, often found in the data, is a common bound on the delta of a call option for all single-factor markov and deterministic volatility function models.

In addition to the implications mentioned above, we also find that investors' posterior beliefs on the true drift rate of dividends play the role of (endogenously generated) non-traded factors, affecting the value of traded assets and hence potentially demanding a market-price of risk. This implies that investors' preference parameters may enter the option pricing formula if it is not plausible to assume that investors know perfectly the underlying drift rate of dividends. Indeed, in our model we recover the Black and Scholes formula when we assume that investors' have perfect information of a constant drift rate of dividends. In this case, investors' beliefs are trivially constant and hence these endogenous non-traded factors vanish.

As far as the methodology of the paper is concerned, we find it useful to discretize the parameter space - i.e. the set of possible drifts for the dividend process - to obtain the dynamics of investors' beliefs in closed form. This approach enables us to fully characterize stock returns, return volatility, and the covariance between returns and volatility. Indeed, we are able to show that the stock's return and volatility depend on a limited number of variables, which depend on investors' estimated drift rate of dividends as well as their confidence in it.

In the special case of a two-state model, we are also able to solve for option prices using Fast Fourier Transform methods. Such methods have been useful for providing analytical results to 
several option pricing problems in recent years, such as those with stochastic jumps, and stochastic volatility (some examples of this methodology are in Heston 1993, Bates 1996, Bakshi et al. 1997, Scott 1997). As in these papers, we are able to reduce the option pricing problem to the numerical Fourier Inversion of the solution of an ordinary differential equation (ODE). The ODE itself has no explicit analytical solution, but its solution can be well approximated by a (small) finite series of polynomials. A numerical Fourier inversion yields the Fourier Transform of the state-return density function. Option prices are then calculated as the sum of the value of four primitive securities, each of which is obtained by evaluating the Fourier Transform at chosen points. The two-state model is also particularly useful in comparative statics exercises. Using our option pricing formula, we study the effects on option prices of changes in the persistence of a good or bad state, in investors' coefficient of risk aversion or in their information quality. We find for example that a higher degree of risk aversion has ambiguous implications for the price of options, because it affects both return volatility and equilibrium interest rates.

After detailing the implications of our model for option prices of the above findings, we also provide a calibration of the model. Real earnings growth on the S\&P 500 for the period 1960:01 - 1998:09 is fitted with a 2-state regime-switching model. The estimation procedure allows us to back out the posterior probabilities of investors on the space of possible drift rates and hence to compute proxies for investors' expected drift rate of earnings, their uncertainty, and the implied theoretical return volatility and covariance between returns and volatility. The latter is shown to be mainly negative over time ( $70 \%$ of the time), with occasional switches to a positive sign. In addition, we provide evidence that the model-generated return volatility, which depends only on the posterior beliefs of investors, can explain the S\&P100 option volatility index. We also test two further predictions of the state-return density function conditional on filtered beliefs using options data. The mean-reversion of the belief process implies that the return-density must be positively (negatively) skewed in periods with low (high) beliefs. This implication is tested by using the ratio of $3 \%$ OTM put-to-call prices. Secondly, the possibility of a large shift in beliefs implies greater kurtosis of the return density function. Therefore the model implies low (high) kurtosis in periods of high (low) uncertainty. For this, we test the implied volatility of a convexity measure of implied volatility, or equivalently, the implied volatility butterfly spread on the filtered belief process. Both tests support the implications of our model. 
There have been several similar learning models in the literature, of which we do not attempt an exhaustive survey. For financial markets with learning in continuous-time Detemple (1986), Dothan and Feldman (1986), and Gennotte (1986) used the results of the Kalman filter to characterize the learning of agents when the unobservable drift of returns followed a diffusion process. This learning paradigm is a useful for understanding several aspects of financial decision making as evident from the recent work of Brennan (1998), who resolves an important assetallocation puzzle by appealing to the role of learning about expected returns. Despite its several advantages, Kalman filtering models imply that the estimation error (the variance of the conditional distribution of agents beliefs) follows a deterministic process that converges to a constant. This property is inherited by the volatility of returns in these models. As an important exception, Yan (1999) recently studied option pricing in a Kalman filtering model where, despite the convergence of estimation error, return volatility was stochastic due to variations in the riskless rate. Also in a Gaussian incomplete information model, but with discrete periodic signals, Duffie and Lando (1999) provide an analysis of the term structure of credit spreads with incomplete accounting information about fundamentals of the firm.

Detemple (1991) used the same assumption on the underlying diffusion process, but assumed that agents started updating with a non-Gaussian prior. In his model, the estimation error indeed exhibited stochastic variation over time, but was characterized by a large number (continuum) of state variables. To exploit the stochastic estimation error of non-Gaussian models, David (1997) studied the asset-allocation problem in a setting where the unobservable drift jumped between a finite number of states. Using a filtering result in Liptser and Shiryaayev (1977), he showed that learning induces the leverage effect (a negative asymmetric relation between returns and future volatility) on the market portfolio, as agents attempt to hedge the risk of changing estimation error. With a single stock and this same filtering problem characterizing the uncertainty about expected dividend growth, Veronesi (1999b) showed that return volatility has GARCH properties and that markets overreact to bad news in good times and underreact to good news in bad times. In an exchange economy, Veronesi (1999a) shows that the volatility of stock-returns in this setting would increase if agents received more precise signals on the unobserved drift. In this paper we study the implications of stochastic estimation error on option prices. ${ }^{3}$

\footnotetext{
${ }^{3}$ In a somewhat similarly motivated paper, Campbell and Li (1999) study option prices in a model of exogenous regime-switching volatility of stock returns. While option prices in their model also depend on beliefs of agents, due to the lack of correlation between returns and volatility, their model implies symmetric implied volatility smiles.
} 
The paper develops as follows: next section introduces the economic model and characterizes investors' beliefs dynamics. In addition, it also characterizes stock prices and the process for stock returns. Section 2 develops the partial differential equation for derivative security pricing when there is uncertainty in the fundamentals. In addition, it draws out the implications on option prices under some assumptions on the "fundamentals." Section 3 derives a closed form solution for options in the special case where there are only two possible states, while Section 4 studies its properties. Section 5 contains the calibration of the model and some empirical tests. Section 6 concludes.

\section{The Model}

Consider an economy populated by a continuum of investors with an iso-elastic utility function

$U(c, t)=e^{-\phi t} \frac{c^{1-\gamma}}{1-\gamma}$. Let $W(t)=\left(W_{1}(t), W_{2}(t), W_{3}(t)\right)^{\prime}$ be a three-dimensional vector of independent Wiener processes. Dividends evolve according to a lognormal process

$$
\frac{d x}{x}=\theta d t+\sigma_{x} d W
$$

where the $1 \times 3$ vector $\sigma_{x}$ is assumed known and constant. The growth rate of dividends $\theta(t)$ is not observable and may move over time according to the process described below. In addition to dividends, investors can infer the value of $\theta(t)$ by observing another public signal described by:

$$
\frac{d e}{e}=\theta d t+\sigma_{e} d W
$$

where again $\sigma_{e}$ is a constant $1 \times 3$ vector. The formulation (2) is analogous to the usual "signal = value plus noise" in the discrete time setting. The choice of a lognormal process for signals is for notational purposes only (see below) and has no substantive effect.

In order to keep the analysis simple, we also assume that the consumption process is exogenously given and follows the process

$$
\frac{d c}{c}=\kappa d t+\sigma_{c} d W
$$


where $\kappa(t)$ is also unobservable and $\sigma_{c}$ is a $1 \times 3$ constant matrix. ${ }^{4}$ This assumption enables us to fully concentrate on the implications of uncertainty and learning on option prices, leaving aside the implications due to market clearing conditions. However, notice that the special case of a Lucas (1978) pure-exchange economy is obtained by setting $c=x, \kappa=\theta$ and $\sigma_{x}=\sigma_{c}$.

To complete the description of the model it is convenient to stack together the "observation" processes $(1),(2)$ and $(3)$ : Let $y=(x, e, c)^{\prime}$, so that

$$
\frac{d y}{y}=\nu(t) d t+\Sigma d W
$$

where $\frac{d y}{y}$ is to be interpreted "element-by-element" division,

$$
\nu(t)=\left(\begin{array}{c}
\theta(t) \\
\theta(t) \\
\kappa(t)
\end{array}\right) ; \quad \Sigma=\left(\begin{array}{c}
\sigma_{x} \\
\sigma_{e} \\
\sigma_{c}
\end{array}\right)
$$

We will assume that the $3 \times 3$ matrix $\Sigma$ is invertible. In order to fully characterize the process for stock returns as well as the movements of investors' posterior distribution over time, it is convenient to discretize the state space for dividend growth and consumption growth. That is to say, we assume that $\theta$ can take only the values in the finite set $\left[\theta_{1}, \ldots, \theta_{n}\right]$ with $n$ possibly very large. Likewise, we assume that consumption growth $\kappa$ can take only values in the finite set $\left[\kappa_{1}, \ldots, \kappa_{n}\right]$. The transition from one state $\nu_{i}=\left(\theta_{i}, \theta_{i}, \kappa_{i}\right)$ to another state $\nu_{j}=\left(\theta_{j}, \theta_{j}, \kappa_{j}\right)$ in the infinitesimal interval $\Delta$ occurs with probability

$$
\lambda_{i j} d t=\operatorname{Pr}\left(v(t+\Delta)=v_{j} \mid v(t)=v_{i}\right)
$$

For later reference, notice that if we let $\iota_{x}=(1,0,0)^{\prime}, \iota_{e}=(0,1,0)^{\prime}$, and $\iota_{c}=(0,0,1)^{\prime}$, we obtain $\sigma_{x}=\iota_{x}^{\prime} \Sigma, \sigma_{e}=\iota_{e}^{\prime} \Sigma, \sigma_{c}=\iota_{c}^{\prime} \Sigma$.

\subsection{The Dynamics of Investors' Beliefs}

Given the observation of $y(t)$, investors form the posterior probability

$$
\pi_{i}(t)=\operatorname{Pr}\left(\nu(t)=\nu_{i} \mid \mathcal{F}(t)\right)
$$

\footnotetext{
${ }^{4}$ The formulation leads to the stochastic discount factor process shown in (8). As a modeling strategy, we could instead specify an exogenous process for the stochastic discount factor. The present specification provides intuition for comparative static experiments of changes in investors' risk-aversion on option prices.
} 
where $\mathcal{F}(t)$ is investors' filtration at time $t$. Let $\pi=\left(\pi_{1}, \ldots, \pi_{n}\right)$ be the vector of beliefs.

Lemma 1 Given an initial condition $\pi(0)=\widehat{\pi}$ with $\sum_{i=1}^{n} \widehat{\pi}_{i}=1$ and $0 \leq \widehat{\pi}_{i} \leq 1$ for all $i$, the probabilities, $\pi_{i}(t)$ satisfy the system of $n$ stochastic differential equations:

$$
d \pi_{i}=\mu_{i} d t+\sigma_{i} d \widetilde{W}
$$

where

$$
\mu_{i}=[\pi \Lambda]_{i} ; \quad \sigma_{i}=\pi_{i}\left(\nu_{i}-\bar{\nu}\right)^{\prime}\left(\Sigma \Sigma^{\prime}\right)^{-\frac{1}{2}} ; \quad \bar{\nu}=\sum_{i=1}^{n} \pi_{i} \nu_{i}=E_{t}\left(\frac{d y}{y}\right)
$$

and

$$
\widetilde{d W}=\left(\Sigma \Sigma^{\prime}\right)^{-\frac{1}{2}}\left(\frac{d y}{y}-E_{t}\left(\frac{d y}{y}\right)\right)=\left(\Sigma \Sigma^{\prime}\right)^{-\frac{1}{2}}(\nu(t)-\bar{\nu}) d t+\left(\Sigma \Sigma^{\prime}\right)^{-\frac{1}{2}} \Sigma d W
$$

Moreover, for every $t>0, \sum_{i=1}^{n} \pi(t)=1$.

Proof: See Liptser and Shiryaayev (1977).

The expressions in (5) are rather intuitive: the drift rate depends only on the prior belief at time $t, \pi(t)$, and the transition matrix $\Lambda$, and therefore the expression describes how the beliefs are updated between $t$ and $t+\Delta$ when no other information is available. The diffusion part in (4) describes the impact on beliefs due to the observation of the vector $y(t+\Delta)$. Specifically, $d \widetilde{W}$ is simply the innovation process normalized by the (square root of) the variance covariance matrix of the observation process $d y / y$. The impact of this normalized expectation error on belief $\pi_{i}$ is described by $\sigma_{i}$ : this tends to be high when there is some uncertainty about the true vector $\nu$. In fact, notice that $\sigma_{i}$ is close to zero for all $i$ when $\pi=\left(\pi_{1}, \ldots, \pi_{n}\right)$ is the degenerate distribution giving probability one to the true value and zero otherwise. Finally, the precision of the signals $d y / y$ affects $d \pi_{i}$ through the variance covariance matrix $\Sigma \Sigma^{\prime}$.

It is worth noticing that

$$
\sum_{i=1}^{n} d \pi_{i}=\sum_{i=1}^{n} \mu_{i} d t+\left(\sum_{i=1}^{n} \sigma_{i}\right) d \widetilde{W}=0
$$

thereby ensuring that for all $t, \sum_{i=1}^{n} \pi_{i}=1$.

For later reference, we rewrite the fundamental process $d y / y$ in terms of the new innovation process $d \widetilde{W}$ in the following corollary: 
Corollary 1 The fundamental process $d y$ follows the process $d y / y=\bar{v} d t+\left(\Sigma \Sigma^{\prime}\right)^{\frac{1}{2}} d \widetilde{W}$, where $d y / y$ should be interpreted as "element-by-element" division.

Proof. In Appendix 1.

Hence, for example, the consumption process with respect to investors' filtration is $\frac{d c}{c}=$ $\bar{k} d t+\iota_{c}\left(\Sigma \Sigma^{\prime}\right)^{\frac{1}{2}} d \widetilde{W}$. Since $d \widetilde{W}$ is an "innovations" process, under the separation principle it can be used for dynamic optimization. See David (1997) for a discussion.

\subsection{Stock Returns and the Risk-Free Rate}

Given investors' utility function, we immediately obtain the stochastic discount factor as the marginal utility of consumption $m(t)=U_{c}(t, c)=e^{-\phi t} c^{-\gamma}$. By Ito's lemma, ${ }^{5}$

$$
d m=\left(-\phi-\gamma \kappa(t)+\frac{1}{2} \gamma(\gamma+1) \sigma_{c} \sigma_{c}^{\prime}\right) m d t-\gamma m \sigma_{c} d W
$$

Hence, the equilibrium stock price $S(t)$ must satisfy the usual pricing relation:

$$
m(t) S(t)=E_{t}\left[\int_{t}^{\infty} m(s) x(s) d s\right]
$$

Proposition 1 (a) For all $i=1, \ldots, n$, let $\widehat{\theta}_{i}=\gamma k_{i}-\theta_{i}+\phi-\frac{1}{2} \gamma(\gamma+1) \sigma_{c} \sigma_{c}^{\prime}+\gamma \sigma_{c} \sigma_{x}^{\prime}>0$ Then, for every $t$, the stock price is given by:

$$
S(t)=x(t) \sum_{i=1}^{n} \pi_{i}(t) C_{i}
$$

where $C_{i}$ 's are positive constants satisfying $\widehat{\theta}_{i} C_{i}=1+\sum_{j=1}^{n} \lambda_{i j} C_{j}$ for all $i=1, \ldots, n$.

(b) The instantaneous risk-free rate is given by $r=\phi+\gamma \bar{\kappa}-\frac{1}{2} \gamma(\gamma+1) \sigma_{c} \sigma_{c}^{\prime}$ where $\bar{\kappa}=\sum_{i=1}^{n} \pi_{i} \kappa_{i}$.

(c) The price of a zero-coupon bond with maturity $\tau$ is

$$
B(t, \tau)=\sum_{i=1}^{n} \pi_{i} B_{i}(\tau)
$$

\footnotetext{
${ }^{5}$ It will be shown later that under investors' information filtration the stochastic discount factor will follow $d m / m=$ $-r d t-\gamma \iota_{c}\left(\Sigma \Sigma^{\prime}\right)^{\frac{1}{2}} d \widetilde{W}$. However, it is convenient for now to leave the process for the stochastic discount factor as in (8).
} 
where $B_{i}(\tau)=E\left(\frac{m(t+\tau)}{m(t)} \mid \nu(t)=\nu_{i}\right)=[\Omega]_{i}\left(e^{w_{1} \tau}, \ldots, e^{w_{n} \tau}\right)^{\prime}$ and where $w_{1}, \ldots, w_{m}$ are the eigenvalues of the matrix $\widehat{\Lambda}=-\Lambda-\gamma \operatorname{diag}(\kappa)-\left(\phi-\frac{1}{2} \gamma(\gamma+1) \sigma_{c} \sigma_{c}^{\prime}\right)$ and $\Omega$ is the matrix of column eigenvectors of $\widehat{\Lambda}$, normalized so that $\sum_{j=1}^{n} \omega_{i j}=1$ for all $i=1, . ., n$.

Proof. In Appendix 1.

To interpret the above result about the stock price, it is shown in the appendix that

$$
C_{i}=\frac{1}{u_{c}(t, c) x(t)} E_{t}\left[\int_{t}^{\infty} u_{c}(s, c) x(s) d s \mid \nu(t)=\nu_{i}\right]
$$

That is, each constant $C_{i}$ represents investors' expectation of future dividends conditional on the state being $\nu_{i}$ today, discounted by their marginal utility of consumption and normalized to make it independent of the current dividend and time. Hence, a high $C_{i}$ implies that investors would be willing to pay a high price relative to the current dividend in state $\nu_{i}$. Since they do not actually observe the state $\nu_{i}$, they weight each $C_{i}$ by its conditional probability $\pi_{i}$ thereby obtaining (10).

Notice from equation (10) the stock price follows a continuous-path process, even if the state space for dividend drift rates is discrete. Indeed, we can easily derive the following proposition:

Proposition 2 The stock return process is given by:

$$
\frac{d S}{S}=\left(\mu_{S}-\delta\right) d t+\sigma_{S} d \widetilde{W}
$$

where

$$
\begin{aligned}
\mu_{S} & =r+\gamma\left(\frac{\sum_{i=1}^{n} \pi_{i} C_{i}\left(\kappa_{i}-\bar{\kappa}\right)}{\sum_{i=1}^{n} C_{i} \pi_{i}}+\sigma_{c} \sigma_{x}^{\prime}\right)=r+\gamma \times \operatorname{Cov}\left(\frac{d c}{c}, \frac{d S}{S}\right) \\
\delta & =\frac{x}{S}=\frac{1}{\sum_{i=1}^{n} C_{i} \pi_{i}} \\
\sigma_{S} & =\left(\iota_{x}\left(\Sigma \Sigma^{\prime}\right)^{\frac{1}{2}}+\frac{\sum_{i=1}^{n} C_{i} \pi_{i}\left(\nu_{i}-\bar{\nu}\right)^{\prime}\left(\Sigma \Sigma^{\prime}\right)^{-\frac{1}{2}}}{\sum_{i=1}^{n} C_{i} \pi_{i}}\right)
\end{aligned}
$$

Proof. In Appendix 1.

This proposition shows that both the stock expected return and its volatility are changing over time, depending on the distribution $\pi=\left(\pi_{1}, \ldots, \pi_{n}\right)$. 


\subsection{Return Volatility and its Covariance with Stock Returns}

In this subsection we characterize the volatility of equilibrium stock returns as well as its covariance with the return process itself. As is well known, these two quantities are extremely important in determining the behavior of option prices. Even though it is not necessary for the result, we limit our investigation to the case where the risk-free interest rate is constant. This will enable us to better compare our option pricing results with Black and Scholes option prices, which also assume a constant interest rate. In addition, this assumption makes sure that our results do not depend on the behavior of an erratic stochastic interest rate. ${ }^{6}$ From proposition 1 a constant interest rate is obtained in our model by assuming $\kappa_{1}=\kappa_{2}=\ldots=\kappa_{n}$.

We start by introducing the following notation: Let

$$
\pi_{i}^{*}=\frac{\pi_{i} C_{i}}{\sum_{j=1}^{n} \pi_{j} C_{j}}
$$

As in Veronesi (1999a), we call $\pi^{*}=\left(\pi_{1}^{*}, \ldots, \pi_{n}^{*}\right)$ the value-weighted probability density: notice that from equation (11) the weights $C_{i}$ are the discounted expected value of future dividends. In this sense, the $\pi_{i}^{*}$ can be thought of as a state-price density function, where the states are $\theta_{1}, \ldots, \theta_{n}$. From now on, a star "*" denotes a quantity computed with respect to the distribution $\pi^{*}$. For example, $\bar{\theta}^{*}$ denotes the mean $\theta$ computed using the distribution $\pi^{*}$. Similarly, let

$$
\sigma_{\theta}^{2}=\sum_{i=1}^{n} \pi_{i}\left(\theta_{i}-\bar{\theta}\right)^{2} ; \quad \sigma_{\theta}^{* 2}=\sum_{i=1}^{n} \pi_{i}^{*}\left(\theta_{i}-\bar{\theta}^{*}\right)^{2}
$$

be the variances of $\theta$ computed using $\pi$ and $\pi^{*}$. We then have:

Proposition 3 Let $\kappa_{1}=\kappa_{2}=\ldots=\kappa_{n}$ and let $b=(1,1,0) \times\left(\Sigma \Sigma^{\prime}\right)^{-\frac{1}{2}}$. Then

(a) Return volatility is given by

$$
V \equiv \sigma_{S} \sigma_{S}^{\prime}=\left(\sigma_{x} \sigma_{x}^{\prime}+2\left(\bar{\theta}^{*}-\bar{\theta}\right)+\left(\bar{\theta}^{*}-\bar{\theta}\right)^{2} b b^{\prime}\right)
$$

(b) Return volatility $V$ follows the process

$$
d V=\mu_{V} d t+\sigma_{V} d W
$$

\footnotetext{
${ }^{6}$ The effects of a stochastic interest rate on option prices in the context of an incomplete information model are explored in Yan (1999).
} 
where

$$
\begin{gathered}
\mu_{V}=\left(\sigma_{\theta}^{* 2}-\sigma_{\theta}^{2}\right)^{2}\left(b b^{\prime}\right)^{2}+2\left(E_{t}\left(d \bar{\theta}^{*}\right)-E_{t}(d \bar{\theta})\right)\left(1+\left(\bar{\theta}^{*}-\bar{\theta}\right) b b^{\prime}\right) \\
\sigma_{V}=\left(\sigma_{\theta}^{* 2}-\sigma_{\theta}^{2}\right)\left(1+\left(\bar{\theta}^{*}-\bar{\theta}\right) b b^{\prime}\right) 2 b,
\end{gathered}
$$

and $E_{t}(d \bar{\theta})$ and $E_{t}\left(d \bar{\theta}^{*}\right)$ are the expected changes in the expected dividend drift rate according to $\pi$ and $\pi^{*}$, respectively. That is,

$$
\begin{aligned}
E_{t}(d \bar{\theta}) & =\left(\sum_{i=1}^{n}[\pi \Lambda]_{i} \theta_{i}\right) d t \\
E_{t}\left(d \bar{\theta}^{*}\right) & =\left(\frac{\sum_{i=1}^{n}[\pi \Lambda]_{i} C_{i}\left(\theta_{i}-\bar{\theta}^{*}\right)}{\sum_{k=1}^{n} \pi_{k} C_{k}}-\sigma_{\theta}^{* 2}\left(\bar{\theta}^{*}-\bar{\theta}\right) b b^{\prime}\right) d t
\end{aligned}
$$

(c) The covariance between return and volatility is given by

$$
\operatorname{Cov}\left(d V, \frac{d S}{S}\right) \equiv \sigma_{V} \sigma_{S}^{\prime}=2\left(\sigma_{\theta}^{* 2}-\sigma_{\theta}^{2}\right)\left(1+\left(\bar{\theta}^{*}-\bar{\theta}\right) b b^{\prime}\right)^{2}
$$

\section{Proof. In Appendix 1.}

This proposition provides a general result on return volatility and its covariance with returns. Importantly, we notice that return volatility is stochastic, it follows a predictable process, and has a stochastic volatility. In particular, the time predictability of volatility has interesting implications for option pricing. Consider the following simple example: Suppose that investors have a very high confidence in a particular state $\theta_{i}$. In this case, from the definition of $\pi^{*}$ we find that $\bar{\theta}^{*} \approx \bar{\theta}$ and similarly $\sigma_{\theta}^{2 *} \approx \sigma_{\theta}^{2}$. From equations (17) and (20), this implies that $V$ is low and the volatility of volatility, $\sigma_{V} \approx 0$. However, if $\Lambda$ is non-zero, from equations (19), (21) and (22) we may have $\mu_{V}$ positive and possibly high. Hence, even though today stock volatility is low, investors expect high volatility in the future. This implies that the implied volatility of option prices is higher than stock volatility.

The previous proposition also shows that the covariance between return and volatility is positive or negative depending on whether the current posterior distribution of beliefs implies that $\sigma_{\theta}^{* 2}$ is greater or smaller than $\sigma_{\theta}^{2}$. That is,

$$
\operatorname{Cov}\left(d V, \frac{d S}{S}\right)<0 \text { if and only if } \sigma_{\theta}^{* 2}<\sigma_{\theta}^{2}
$$


We can get intuition about this result by looking at the simple two-state case. In this case, the return volatility $V$ can be plotted as a function of the posterior probability of being in the high state $\pi_{2}$. We plot $\sqrt{V}$ in Figure 5 for parameter values calibrated to S\&P 500 real earnings growth. Consider now the case where the probability of being in the high state, $\pi_{2}$, is close to one. In this case, $\bar{\theta}$ is high and $\sigma_{\theta}^{2}$ is small. A series of negative innovations in the dividend process decreases the stock prices thereby generating negative returns. At the same time, it also decreases $\pi_{2}$ and hence increases return volatility $V$ (as well as the level of uncertainty $\sigma_{\theta}^{2}$ ). Since volatility increases with negative returns, we have a negative covariance of return volatility and stock returns for $\pi_{2}$ high. The opposite argument illustrates that when $\pi_{2}$ is low we have a positive covariance between return volatility and stock returns.

We make the above argument formal in the following corollary:

Corollary 2 Let $\kappa_{1}=\kappa_{2}$. Then, $V$ is a strictly concave function of $\pi \equiv \pi_{2}$ and $\operatorname{Cov}\left(d V, \frac{d S}{S}\right)<$ $0 \quad$ if and only if $\pi>\hat{\pi}$ where $\hat{\pi}=\operatorname{argmax}_{\pi} V$.

Proof. In Appendix 1.

\section{The Value of Contingent Claims}

In this section we determine the value of a contingent claim written on the stock price $S$. Since the evolution of $S$ is non-Markovian, we need to add all the state variables (i.e. beliefs) $\pi_{-n}=$ $\left(\pi_{1}, \ldots, \pi_{n-1}\right)$ in order to describe the economy. Another appeal to the Separation Principle (c.f., for example David 1997) implies that the system $\left(S, \pi_{1}, \ldots, \pi_{n-1}\right)$ is Markovian. ${ }^{7}$ Let $f\left(t, S, \pi_{-n}\right)$ denote the value at time $t$ of the contingent claim, when the price of the stock is $S$ and beliefs are represented by the vector $\pi$

Proposition 4 Each security $f\left(t, S, \pi_{-n}\right)$ satisfies the following PDE:

$$
\begin{aligned}
r f= & \frac{\partial f}{\partial t}+\frac{\partial f}{\partial S} S\left(r-\frac{1}{\sum_{i=1}^{n} C_{i} \pi_{i}}\right)+\sum_{j=1}^{n-1} \frac{\partial f}{\partial \pi_{j}}\left(\mu_{j}-\gamma \pi_{j}\left(\kappa_{j}-\bar{\kappa}\right)\right)+\frac{1}{2} \frac{\partial^{2} f}{\partial S^{2}} S^{2} \sigma_{S} \sigma_{S}^{\prime} \\
& +\sum_{j=1}^{n-1} \frac{\partial^{2} f}{\partial S \partial \pi_{j}} S \sigma_{S} \sigma_{i}^{\prime}+\frac{1}{2} \sum_{k=1}^{n-1} \sum_{j=1}^{n-1} \frac{\partial f^{2}}{\partial \pi_{k} \partial \pi_{j}} \sigma_{k} \sigma_{j}^{\prime}
\end{aligned}
$$

where $\sigma_{j}$ is given by (5).

\footnotetext{
${ }^{7}$ Indeed, the system $\left(S, \pi_{1}, \ldots, \pi_{n-1}\right)$ is Markovian because we always have $\pi_{n}=1-\sum_{j=1}^{n-1} \pi_{j}$.
} 


\section{Proof. In Appendix 1.}

This proposition shows that the price of any derivative security depends on investors' posterior distribution $\pi=\left(\pi_{1}, \ldots, \pi_{n-1}\right)$. Moreover, each probability $\pi_{i}$ has a market price of risk given by:

Corollary 3 The market price of risk of belief $\pi_{i}$ is given by:

$$
\gamma \pi_{i}\left(\kappa_{i}-\bar{\kappa}\right)=\gamma \operatorname{Cov}\left(\frac{d c}{c}, d \pi_{i}\right)
$$

Proof. In Appendix 1.

It is interesting to notice that the market price of risk of each "belief" $\pi_{i}$ is positive or negative depending on whether the consumption drift rate $\kappa_{i}$ is above or below the expected consumption drift $\bar{\kappa}$. The assumption of an exogenously given constant and known consumption growth can be modeled in this framework by assuming that $\kappa_{i}=\kappa_{j}=\bar{\kappa}$. In this case, beliefs have zero covariance with consumption because the latter is exogenous and hence there is zero market price of $\pi_{i}$. Before commenting further on equation (25) consider the following:

Corollary 4 Under perfect information and no changes in state, the Black and Scholes option pricing formula obtains.

Corollary 4 is quite straightforward. In this case in fact, we have that the transition matrix $\Lambda=0$ and that for all $t>0, \pi_{k}=1$ for some $k$ and $\pi_{j}=0$ for all $j \neq k$. Hence, the price of the asset is $S(t)=x(t) C_{k}$ and therefore all the parameters $\mu_{S}=r+\gamma \sigma_{x} \sigma_{c}^{\prime}, \delta=1 / C_{k}, \sigma_{S}=\sigma_{x}$ and $r=\phi+\gamma \kappa_{k}-\frac{1}{2} \gamma(\gamma+1) \sigma_{c} \sigma_{c}^{\prime}$ are constant. Since we are in the Black and Scholes setup we see that (24) turns to the standard PDE

$$
r f=\frac{\partial f}{\partial t}+\frac{\partial f}{\partial S} S(r-\delta)+\frac{1}{2} \frac{\partial^{2} f}{\partial S^{2}} \sigma_{S} \sigma_{S}^{\prime} S^{2}
$$

Notice that in the case of perfect information and constant underlying true state $(\Lambda=0)$ we obtain the usual result that contingent claims do not depend on investors' preferences. This is to be contrasted with the result in (24) that shows that this is not generally the case when there is uncertainty. Indeed, equation (24) holds true even if $\Lambda=0$, as is assumed in corollary 4. Hence, the introduction of incomplete information in a model with constant parameters has the effect of 
endogenously generating a set of state variables $\pi_{i}$, each of which carries its own market price of risk given by (25).

We end the discussion of this subsection with two remarks: first, we restate the processes of stock returns and state variables under the risk-neutral measure and second, we rewrite the return process under the assumption that dividends are continuously reinvested in the stock itself. Since the latter can be considered a security with no dividends, comparison with standard models will be easier.

Corollary 5 The risk neutral processes for stocks $S$ and beliefs $\pi_{i}$ are given by:

$$
\begin{aligned}
\frac{d S}{S} & =(r-\delta) d t+\sigma_{S} d \widetilde{W}^{*} \\
d \pi_{i} & =\left(\mu_{i}-\gamma \pi_{i}\left(\kappa_{i}-\bar{\kappa}\right)\right) d t+\sigma_{i} d \widetilde{W}^{*}
\end{aligned}
$$

where $\widetilde{W}^{*}$ is a three-dimensional Wiener process under the risk-neutral measure.

Proof. In Appendix 1.

Notice that the risk-neutralized probabilities in (28) still share the property that for all $t, \pi(t) \in[0,1]$ and $\sum_{i=1}^{n} d \pi_{i}=0$, as it was true in equation (7).

Corollary 6 Let $\widehat{S}$ be the value of the portfolio with $N$ shares, where $N$ grows according to $d N=$ $N \frac{x}{S} d t$ Then, $d \widehat{S} / \widehat{S}=\mu_{S} d t+\sigma_{S} d \widetilde{W}$ where $\mu_{S}$ and $\sigma_{S}$ are defined in (12) and (14).

Proof. In Appendix 1.

\subsection{Uncertainty, Risk aversion, Volatility and Option Prices}

In this subsection we provide further results of comparative statics for stock return volatility and discuss their implications for option prices. Specifically, we investigate how the precision of investors' information on the true drift of dividends and their degree of risk aversion affect stock returns and hence option prices. These results will be useful to interpret the numerical findings in the subsequent sections. As before, we concentrate our attention to the case where the interest rate is constant, that is $\kappa_{1}=\kappa_{2}=\ldots=\kappa_{n}$.

We now characterize the quantities in proposition 3 under the assumption that the constants $C_{i}$ 's are either increasing in $\theta_{i}$ or increasing and convex in $\theta_{i}$. The first is quite a natural 
assumption implying that a higher expected growth rate of dividends yields a higher price-dividend ratio. The second one, that is, $\frac{C_{i}-C_{i-1}}{\theta_{i}-\theta_{i-1}}<\frac{C_{i+1}-C_{i}}{\theta_{i+1}-\theta_{i}}$, implies that a $1 \%$ increase in expected dividends increases the price-dividend ratio by more than $1 \%$. This occurs for example in the following cases:

I Constant Unknown dividend drift: Investors do not know the value of $\theta(t)$ which is assumed to be constant and that can take any of the values $\theta_{1}<\theta_{2}<\ldots<\theta_{n}$. Formally, this implies that $\Lambda=0$. Solving for the constants in proposition 1 (a) we can see immediately that $C_{i}$ 's satisfy the above assumptions.

II State-independent probability of switching: This is the same as case I, but with the assumption that $\lambda_{i j}=\widehat{\lambda}_{j}$, for $j \neq i$ and $\lambda_{i i}=-\sum_{j \neq i} \widehat{\lambda}_{j}=\widehat{\lambda}_{i}-\sum_{j=1}^{n} \widehat{\lambda}_{j}$, where $\widehat{\lambda}_{1}, \ldots, \widehat{\lambda}_{n}$ are jump intensities. From proposition 1 it is again possible to show that $C_{i}$ 's satisfy the above assumptions. ${ }^{8}$

III Two-state model: This case will be investigated in more detail in the next section.

We first state a useful Lemma:

Lemma 2 Let $\kappa_{1}=\kappa_{2}=\ldots=\kappa_{n}$. Suppose that the constants $C_{i}$ are increasing in $\theta_{i}$. Then for any distribution $\pi$ we have $\bar{\theta}^{*}=\sum_{j=1}^{n} \pi_{j}^{*} \theta_{j}>\sum_{j=1}^{n} \pi_{j} \theta_{j}=\bar{\theta}$

We then have:

Proposition 5 (a) If $C_{i}$ 's are increasing, then return volatility is always greater than the dividend volatility. That is, $V=\sigma_{S} \sigma_{S}^{\prime}>\sigma_{x} \sigma_{x}^{\prime}$

(b) Given $\pi=\left(\pi_{1}, \ldots, \pi_{n}\right)$, if $C_{i}$ are increasing and convex and if $C_{i} \times\left(\theta_{i}-\theta_{n}\right)$ is also convex, then a mean preserving spread on $\pi$ increases the stock return volatility $V=\sigma_{S} \sigma_{S^{\prime}}$ and decreases the dividend yield $\delta .9$

(c) For given $\pi=\left(\pi_{1}, \ldots, \pi_{n}\right)$, if $C_{i}$ are increasing and convex, a decrease in the signal's diffusion $\sigma_{e 2}$ increases the stock return volatility $V=\sigma_{S} \sigma_{S^{\prime}}$ and but does not affect the dividend yield $\delta$.

\footnotetext{
${ }^{8}$ Indeed, one can verify that defining the constant $K=\frac{\sum_{i=1}^{n} \widehat{\lambda}_{i}}{G(\gamma)-\theta_{i}+\sum_{j=1}^{n} \widehat{\lambda}_{j}}$ where $G(\gamma)$ is defined in proposition 6 we have $C_{i}=1 /\left[\left(G(\gamma)-\theta_{i}+\sum_{j=1}^{n} \widehat{\lambda}_{j}\right)(1-K)\right]$.

${ }^{9} A$ mean-preserving spread of the distribution $\pi$ is given by $\widehat{\pi}$ defined by $\widehat{\pi}_{i}=\pi_{i}+s_{i}$ where for $i_{1}<i_{2}<i_{3}<i_{4}$, $s_{i_{1}}=-s_{i_{2}}=\alpha>0, s_{i_{4}}=-s_{i_{3}}=\beta>0, s_{i}=0$ otherwise, and such that $1>\widehat{\pi}_{i}>0$ and $\alpha\left(\theta_{i_{1}}-\theta_{i_{2}}\right)=\beta\left(\theta_{i_{3}}-\theta_{i_{4}}\right)$. Intuitively, a mean-preserving spread moves probability mass from the "center" of the distribution towards its "tails" without changing the mean (see e.g. Ingersoll (1987))
} 
Proof. In Appendix 1.

The results of the above proposition suggest the following conjectures about the properties of option prices. These will be confirmed by numerical examples in the next section.

Implication 1. Option prices will generally be higher in an economy with uncertain dividend drift rate, compared to an identical economy with a constant and observable dividend growth. In fact, in the latter case we know from the discussion after corollary 4 that return volatility is equal to the dividend volatility.

Implication 2. A mean preserving spread on investors' posterior distribution increases call prices but it has an ambiguous effect on put prices (because of the decrease in the dividend yield). In the case of reinvested dividends both calls and puts increase in value. Notice that the additional condition required in point (b) of the proposition is satisfied in the examples (I) - (III) mentioned above.

Implication 3. Given an initial distribution $\pi=\left(\pi_{1}, . ., \pi_{n}\right)$, an increase in the precision of the signal increases option prices. This seemingly contradictory implication stems from the fact that we are keeping the original $\pi$ constant. Of course, more precise signals will tend to generate more concentrated posteriors on average (and hence lower option prices on average due to Implication 2). The intuitive reason why (c) in proposition 5 holds is the following: consider the extreme case where today we do not know whether $\theta=\theta_{1}$ or $\theta=\theta_{2}$ but in an instant (after we buy the option) we will discover its value perfectly. In this case, in the next instant the stock price will have a jump in price to adjust to the new value of the dividend growth rate. Hence, it is going to have an instantaneous infinite volatility.

Finally, in order to characterize the relationship between the coefficient of risk aversion $\gamma$, the stock return volatility, and the dividend yield, we need to be more specific about the constants $C_{i}$ 's. Specifically, the next proposition holds for cases (I)-(III) above:

Proposition 6 Define $G(\gamma)=\phi+\gamma \kappa-\frac{1}{2} \gamma(\gamma+1) \sigma_{c} \sigma_{c}^{\prime}+\gamma \sigma_{c} \sigma_{x}^{\prime}=r+\gamma \sigma_{c} \sigma_{x}^{\prime}$. In cases (I)-(III) above, if $G^{\prime}(\gamma)>0$, then an increase in the coefficient of risk aversion $\gamma$ decreases return volatility and increases the dividend yield.

Proof In Appendix 1. 
Implication 4. This proposition implies that an increase in the coefficient of risk aversion has ambiguous effects on option prices. In fact, in general an increase in $\gamma$ increases the interest rate, decreases volatility and increases the dividend yield. While the first effect increases a call option price, the latter two decrease it. Similarly, while the first two decrease a put option price, the latter increases it. In the case of reinvested dividends put option prices decrease with risk aversion.

\section{Option Prices in the Two-State Case}

In this section we discuss in more detail the case where the expected fundamental growth rate (drift) can only assume two values. The two-state model has several advantages: first, since the model has low dimensionality, it is possible to solve for option prices using Fast Fourier Transform methods (some examples of this methodology are in Stein and Stein 1991, Heston 1993, Bates 1996, Bakshi et al. 1997, Scott 1997). Second, it is possible to provide intuitive examples of the general results about stock return volatility which were obtained in the previous section.

For notational convenience, let $A=C_{2}-C_{1}, B=C_{1}$ and $b=\left(v_{2}-v_{1}\right)\left(\Sigma \Sigma^{\prime}\right)^{-\frac{1}{2}} \cdot{ }^{10}$ We will denote by $\pi=\pi_{2}$ the only state variable, which fully describes investors' beliefs. In this case, the volatility of stock returns, $V$, is given by

$$
V=\left(\sigma_{x} \sigma_{x}^{\prime}+\frac{A^{2} \pi^{2}(1-\pi)^{2} b b^{\prime}}{(\pi A+B)^{2}}+2 \frac{A \pi(1-\pi)\left(\theta_{2}-\theta_{1}\right)}{\pi A+B}\right)
$$

and the PDE reduces to

$$
\begin{aligned}
r f= & \frac{\partial f}{\partial t}+\frac{\partial f}{\partial S} S\left(r-\frac{1}{A \pi+B}\right)+\frac{\partial f}{\partial \pi}\left(\left(\lambda_{12}+\lambda_{21}\right)\left(\pi^{*}-\pi\right)-\pi(1-\pi) \gamma\left(\kappa_{2}-\kappa_{1}\right)\right)+ \\
& +\frac{1}{2} \frac{\partial^{2} f}{\partial S^{2}} S^{2}\left(\sigma_{x} \sigma_{x}^{\prime}+\frac{A^{2} \pi^{2}(1-\pi)^{2} b b^{\prime}}{(\pi A+B)^{2}}+2 \frac{A \pi(1-\pi)\left(\theta_{2}-\theta_{1}\right)}{\pi A+B}\right)+ \\
& +\frac{\partial^{2} f}{\partial S \partial \pi} S\left(\frac{\pi^{2}(1-\pi)^{2} A b b^{\prime}}{\pi A+B}+\pi(1-\pi)\left(\theta_{2}-\theta_{1}\right)\right)+\frac{1}{2} \frac{\partial^{2} f}{\partial \pi^{2}} \pi^{2}(1-\pi)^{2} b b^{\prime}
\end{aligned}
$$

The price of a call option can be obtained as follows:

Proposition 7 The call option price can be unbundled into a portfolio of Arrow-Debreu securities, each of which can be recovered from the Fourier transform of the state return density conditional

\footnotetext{
${ }^{10}$ Notice the slightly different notation for $b$ compared to the previous section. This is to avoid introducing further notation in the paper.
} 
on $z_{t}=\log \left(S_{t}\right), \bar{f}\left(\omega_{1}, \pi_{t}, T-t ; z_{t}\right)$, by numerical inversion. The call option price is

$$
C^{U}\left(z_{t}, K, \pi, T-t\right)=G\left(\pi_{t}, T-t ; z_{t}\right) \cdot \Pi_{1}\left(z_{t}, K, \pi_{t}, T-t\right)-B\left(\pi_{t}, T-t\right) \cdot K \cdot \Pi_{2}\left(z_{t}, K, \pi_{t}, T-t\right),
$$

$B\left(\pi_{t}, T-t\right)$ is the time-t price of the discount bond maturing after $T-t$ periods, $G\left(\pi_{t}, T-t ; z_{t}\right)$ is the time-t price of a commitment to deliver a unit of stock at time $T$, and is hence the forward price for a unit of stock, and $\Pi_{1}\left(z_{t}, K, \pi_{t}, T-t\right)$ and $\Pi_{2}\left(z_{t}, K, \pi_{t}, T-t\right)$ are, respectively, the time- $t$ Arrow-Debreu securities that pay a dollar if the option finishes in the money under two different measures. The discount bond price and the forward price are given by

$$
\begin{aligned}
B\left(\pi_{t}, T-t\right) & =\bar{f}\left(0, \pi_{t}, T-t ; z_{t}\right) \\
G\left(\pi_{t}, T-t ; z_{t}\right) & =\bar{f}\left(1 / i, \pi_{t}, T-t ; z_{t}\right),
\end{aligned}
$$

and can be obtained by simply evaluating the Fourier Transform at two different points. An $M$ term polynomial approximation of the Fourier Transform $\bar{f}^{M}\left(\omega_{1}, \pi, T-t ; z_{t}\right)$ is in (6) of Appendix 2.

Proof. See Appendix 2.

To perform the inversion as well as the computation of option prices we define the following functions as in Scott (1997):

$$
\begin{aligned}
g_{1}\left(\omega_{1}, \pi, T-t ; z_{t}\right) & =\bar{f}\left(1 / i+\omega_{1}, \pi_{t}, T-t ; z_{t}\right) / \bar{f}\left(1 / i, \pi_{t}, T-t ; z_{t}\right) \\
g_{2}\left(\omega_{1}, \pi_{t}, T-t ; z_{t}\right) & =\bar{f}\left(w_{1}, \pi_{t}, T-t ; z_{t}\right) / \bar{f}\left(0, \pi_{t}, T-t ; z_{t}\right)
\end{aligned}
$$

Then, by the Fourier Inversion formula (c.f., for example, Kendall and Stuart 1977) the probability distribution functions can be written as

$$
\Pi_{j}\left(z_{t}, K, \pi_{t}, T-t\right)=\frac{1}{2}+\frac{1}{\Pi} \int_{0}^{\infty} \operatorname{Re}\left[\frac{e^{-i \omega_{1} \log K} g_{j}\left(\omega_{1}, \pi_{t}, T-t ; z_{t}\right)}{i \omega_{1}}\right] d \omega_{1}
$$

Theorem 1 in Bakshi and Madan (1999) shows that the two p.d.f.s, $\Pi_{1}$, and $\Pi_{2}$ are equivalent to the value of the Arrow-Debreu securities that pay a dollar if the stock price exceeds the exercise price under two different measures. 
For an M-term approximation to the ordinary differential equation (ODE) (47) and its subsequent Fourier inversion, we must replace $\bar{f}\left(\omega_{1}, \pi_{t}, T-t ; z_{t}\right)$ by its M-term approximation $\bar{f}^{M}\left(\omega_{1}, \pi_{t}, T-t ; z_{t}\right)$ and subsequently compute the M-term option price. In our numerical exercises we have found that convergence of option prices occurs rapidly, and $M=6$ is generally sufficient for accuracy up to 4 decimal places for option prices on an underlying stock price of $\$ 100$ (all prices reported in the paper have been verified by Monte-Carlo simulations).

The call option pricing formula in (31) and the analogous formula for puts are convenient enough to provide various partial-derivatives of call option prices with little additional numerical computation. Later, in Section 5 we shall study the hedging properties of U-options; in the following Corollary we provide simple formulas for M-term approximations of partial derivatives with respect to the stock price and beliefs.

Corollary 7 The delta of the call option price in Proposition 7 with respect to the stock price, $C_{S}$, and beliefs, $C_{\pi}$, are given by:

$$
\begin{aligned}
C_{S}^{U}\left(z_{t}, K, \pi, T-t\right) & =\frac{G\left(\pi_{t}, T-t ; z_{t}\right)}{S}\left[\Pi_{1}\left(z_{t}, K, \pi_{t}, T-t\right)+\Pi_{1 z}\left(z_{t}, K, \pi_{t}, T-t\right)\right] \\
& -\frac{K}{S} B\left(\pi_{t}, T-t\right) \Pi_{2 z}\left(z_{t}, K, \pi_{t}, T-t\right) \\
C_{\pi}^{U}\left(z_{t}, K, \pi, T-t\right) & =G_{\pi}\left(\pi_{t}, T-t ; z_{t}\right) \Pi_{1}\left(z_{t}, K, \pi_{t}, T-t\right)+G\left(\pi_{t}, T-t ; z_{t}\right) \Pi_{1 \pi}\left(z_{t}, K, \pi_{t}, T-t\right) \\
& -K \cdot\left[B\left(\pi_{t}, T-t\right) \Pi_{2 \pi}\left(z_{t}, K, \pi_{t}, T-t\right)+B_{\pi}\left(\pi_{t}, T-t\right) \Pi_{2}\left(z_{t}, K, \pi_{t}, T-t\right)\right],
\end{aligned}
$$

where the partial derivatives $\Pi_{i z}, \Pi_{i \pi}, i=1,2, G_{\pi}, G_{z}$, and $B_{\pi}$ are provided in the proof in Appendix 2.

Proof. See Appendix 2.

\section{Option Pricing Results}

In this section we present some of the relationships between different parameters of the model and their effects on option prices. We find it useful to analyze the patterns of option prices over the belief space in terms of three basic factors: 1) the volatility of asset prices, 2) the dividend yield, and 3) the mean reversion of beliefs. It can be easily shown that the shapes of 1) and 2) are similar across different parameter values, and examples are shown in the top panels of Figure 5. As evident from the top right panel, the volatility is alway highest near $1 / 2$; actually as evident to the 
discerning eye, volatility reaches its maximum slightly to the left of $1 / 2$. The size of the asymmetry will depend on the parameters. The dividend yield, $\delta=1 /(\pi A+B)$, is always monotonically declining in $\pi$. These two factors will completely capture the pattern of option prices for 'short' maturities. To separate out the two effects, we will also study the properties of option prices for the same stock, but with dividends reinvested. In solving for the PDE, we just set $\delta=0$.

The mean reverting properties of the belief process are parsimoniously captured in the stationary distribution over the belief space of agents — which is the long-run distribution over beliefs. If it exists, a stationary density $\Psi(x)$ of a diffusion process $X_{t}$ necessarily satisfies:

$$
\Psi(y)=\int \Psi(x) \cdot p(t, x, y) d x, \quad \forall t>0
$$

where, $p(t, x, y)$ is the transition density function i.e., $P(t, x, y)=\operatorname{Prob}\left(X_{t} \leq y \mid X_{0}=x\right)$ and $\frac{d P(t, x, y)}{d y}=p(t, x, y)$.

Lemma 3 The stationary distribution over beliefs under the risk-neutral measure is given by

$$
\Psi(\pi)=\exp \left(\frac{-2 \cdot\left(\lambda_{12} \pi+\lambda_{21}(1-\pi)\right)}{b \cdot b^{\prime} \cdot \pi(1-\pi)}\right) \cdot\left(\frac{1-\pi}{\pi}\right)^{\frac{2 \cdot\left(\gamma \cdot\left(\kappa_{1}-\kappa_{2}\right)+\lambda_{12}-\lambda_{21}\right)}{b \cdot b^{\prime}}} \cdot \frac{1}{b \cdot b^{\prime} \cdot(1-\pi)^{2} \cdot \pi^{2}}
$$

where $b=\left(\theta_{1}-\theta_{2}, \theta_{1}-\theta_{2}, \kappa_{1}-\kappa_{2}\right)^{\prime}\left(\Sigma \Sigma^{\prime}\right)^{-\frac{1}{2}}$, and $C_{1}$ is a constant that ensures that the density integrates to 1. The stationary distribution of beliefs under the true measure is obtained by setting $\kappa_{1}=\kappa_{2}$ in (34).

The shape of the stationary distribution depends on the characteristics of the learning process; some examples will be provided in the subsections below.

In each of the subsections 4.1 to 4.3 , we examine the effects of varying one parameter of the model. We look at the prices of at-the-money call options with a strike price of $\$ 100$ and six months to maturity. In each case, we study the variation of implied volatility of the $C^{\mathrm{U}}\left(z_{0}, K, \pi, T-t\right)$ price as given in (31) as backed out by an econometrician who does not observe beliefs, but instead finds a level of volatility that justifies the Black-Scholes price, when it is assumed that the econometrician uses the unconditional average of the dividend-yield, $\delta^{*}=\int_{0}^{1} 1 /(\pi A+B) \cdot \Psi(\pi)$. Subsection 4.4 instead investigates the properties of implied volatilities across strike prices ("smile effects").

Unless noted, we always assume that the interest rate $r$ is constant, which is achieved by setting $\kappa=\kappa_{1}=\kappa_{2}$ in proposition 1 (b). This also implies that investors know the consumption drift 
rate. In each case, we also compare the implied volatility of U-options with the benchmark of the unconditional average volatility $\sigma^{*}=\int_{0}^{1} \sigma(\pi) \cdot \Psi(\pi)$, which would be implied by the econometrician if the Black-Scholes (B-S) assumptions were indeed true.

\subsection{Information Quality and Option Prices}

We first examine how the precision of the signals on the true drift rate of dividends affects option prices. When $\sigma_{e 2}$ is small, investors' have good information about the true drift rate and hence they "learn fast" the true drift. In this case, the stationary density $\Psi(\pi)$ tends to be double-humped over beliefs. When instead $\sigma_{e 2}$ is high it takes them longer to learn about $\theta(t)$, leading to a single mode, inverse U-shape stationary distribution over beliefs. Examples of these are shown in the top two panels of Figure 2. It is worth mentioning that also the frequency of switches affects the shape of the stationary distribution: High values of $\lambda_{i j}$ imply frequent switches and hence a stationary distribution similar to the one for the "slow learning" case while low values of $\lambda_{i j}$ imply a stationary distribution similar to the one for the "fast learning" case.

We study cases of options on stocks with reinvested dividends, and with distributed dividends. In each of the two bottom panels, the implied volatility is higher for the faster learning case, due to the higher volatility that results from (17). Indeed, this is consistent with the result in Proposition 5 and Implication 3 discussed right after it. As seen in the left panel, the maximum implied volatility occurs to the left of $1 / 2$ for each case, although the effect is larger for the faster learning model. Option value are greater to the left of $1 / 2$, reflecting the asymmetry in volatility. The difference in the implied volatilities is the largest near the maximum volatility. In the right panel, the case of distributed dividends, we notice that the asymmetry in implied volatility almost disappears because the left asymmetry of stock volatility is compensated by the asymmetry to the right due to the dividend yield effect. From Figure 5, it is evident that the dividend yield is always declining in $\pi$, therefore boosting call option prices for a belief of the high state larger than $1 / 2$.

\subsection{Asymmetry of Fundamentals' Growth Rates and Option Prices}

In this subsection we examine the effects on option prices when switching probabilities out of the high and low growth rate states are not symmetric. We will see that the mean-reversion of beliefs in such cases are more rapid than for symmetric growth rate stocks. Keeping the same parameters as before, we set switching parameters for the three cases displayed in Figure 3. In each case the 
average number of switches in each stock fundamental is constant. The middle panel is similar to the slower learning case in subsection 4.1. The stationary densities on the left panels show that the mass is concentrated near 1, for the 'Persistent High Growth' case, and near 0, for the 'Persistent Low Growth' case.

We first notice that option prices for the low persistent case (top panel) are much higher to the right of $1 / 2$. This happens because when $\pi$ is larger than $1 / 2$, it is anticipated that beliefs will return to the mode soon, taking the stock through a period of high volatility. The exact opposite is true for the persistent high growth state - the bottom panel — where beliefs are pulled from the left to the right, thus causing higher option prices to the left of $1 / 2$. The intermediate case of symmetric growth rates have close to (but not exactly) symmetric implied volatilities. Also we note that the difference between the conditional implied volatilities and the unconditional volatility (B-S implied volatility) is large for the asymmetric cases, conditional on beliefs being away from the modal beliefs. This results because of the more rapid reversion to mode in these situations, causing rapid changes in volatility and dividend yields, and hence gives U-option prices the effects observable in models with autocorrelated volatility and jumps.

\subsection{Risk-Aversion and Option Prices}

Since the risk aversion parameter $\gamma$ enters also the market price of belief-risk (see corollary 3 ), it is interesting to study the two following cases: (I) Constant drift of consumption growth, $\kappa=\kappa_{1}=\kappa_{2}$. This is the same case studied in all the other examples in this section, and implies a zero market price of belief-risk and a constant interest rate as shown in proposition 1. (II) Unobserved and state-dependent drift of consumption growth, with $\kappa_{2}>\kappa_{1}$. In this case, the market price of beliefrisk is non-zero and hence the belief process under the risk-neutral measure provided in equation (28) will be used for the pricing of options.

For case (I) we find that in general, a higher risk-aversion parameter for the model leads to lower implied volatilities on $C^{\mathrm{U}}$ options, even though they lead to higher option prices. Implied volatility for two levels of riskaversion are shown in the top panels of Figure 4, which have parameter corresponding to the slow, symmetric learning case. In addition, $\gamma=2$ in the left panel and $\gamma=10$ in the right panel. As it was shown in Proposition 6 and discussed in Implication 4, the volatility function (17) implies that volatility is actually decreasing in risk-aversion. Nonetheless, both the 
dividend yield and the risk-free rate increase with higher risk aversion. On balance, option prices do increase (not shown), but because the BS benchmark increases more, implied volatilities decrease.

For Case (II), we find that the effect of unobservable drift of consumption growth is to introduce an asymmetry in the implied volatilities and stationary densities over the belief space. As seen in the middle and right panels, the stationary density has more mass to the left of $\pi^{*}$, which equals $1 / 2$ for the symmetric case being considered, while implied volatilities are higher to the right of $\pi^{*}$. The effect is more pronounced for the higher risk-aversion case (in fact for $\gamma=2$, the effect is barely visible). The intuition is as follows: The drift of the process under the true measure is $\left(\lambda_{12}+\lambda_{21}\right) \cdot\left(\pi^{*}-\pi\right)$, which is positive for $\pi<\pi^{*}$, and negative for $\pi>\pi^{*}$; under the risk-neutral measure this drift is reduced by the market price of risk, $\gamma \cdot\left(\kappa_{2}-\bar{\kappa}\right)$, as shown in Corollary 3. Therefore, the drift under the risk-neutral measure is less positive for $\pi<\pi^{*}$, and more negative for $\pi>\pi^{*}$, resulting in a process that reverts less strongly for low beliefs and more strongly for high beliefs and causes the stationary density to have more mass to the left of $\pi^{*}$. Implied volatilities become higher to the right of $\pi^{*}$, because of the stronger pull towards $\pi^{*}$, which is close to $\hat{\pi}$, the point of highest volatility.

\subsection{Implied Volatility Across Strike Prices - Asymmetric Smiles and Frowns}

We finally study the behavior of implied volatility across different strike prices for persistent high growth rate stocks, such as the model calibrated to real earnings growth on the S\&P 500 in Section 5. The estimated parameters of the earnings growth process are in Table 1. Figure 5 displays four variables - the endogenous dividend yield, return volatility, stationary density, and the implied volatility for 6 -month at-the-money call options - each as a function of the agent's belief $\pi$. To study the full implications for option prices we characterize the density and the moments of 2month returns for three initial beliefs, $\pi=0.1,0.5,0.9$, in Figure 6 . The density and moments are easily obtained by inverting the Fourier Transform of the state return density function given by $\bar{f}_{r}^{M}=\sum_{n=0}^{M} b_{n}\left(\omega_{1}, .167\right) \cdot(\pi-.5)^{n}$, and the $b_{n}$ are in (51) in Appendix 2. The left-hand side panels display the state-return density functions conditional on three different initial beliefs $\pi=0.9$ (top panel), $\pi=0.5$ (center panel) and $\pi=0.1$ (bottom panel). For each probability $\pi$, we also show for comparison a Gaussian density with the same first and second moments as of the return process of the uncertainty model. The right panel plots the implied volatility across strike prices (smile/frown) for each of these cases. 
The return densities for the three cases are markedly different: for a high $\pi$, the return density exhibits a fat left tail, for a low $\pi$, the density has a fat right tail, and for the medium beliefs, the the density is bi-modal with larger mass on the right hump. The immediate implication of these densities is a negatively (positively) sloped implied volatility smirk for the high (low) belief case and a frown for the medium belief case. Some testable time-series properties of the densities will be examined in detail in Section 5. Here we simply note that, based on the stationary density in Figure 5 , the calibrated model implies a smirk of either direction for about four-fifths of all observations. The model also implies a flip in the sign of the smirk as beliefs cross $1 / 2$. For beliefs close to $1 / 2$, the model implies a frown with a bi-modal density. Indeed, bimodal state price density functions and frowns have been observed in the data, even though they are not so common; as Shimko (1993) writes

Using our methodology, we were able to observe several instances of two-humped [stateprice] distributions for the OEX in the late 1980s; they have since become rare.

The intuition underlying these findings is quite straightforward. The two state model implies that earnings growth is generated by a mixture of distributions, where the exact mixture at a point in time is determined by the beliefs of the agent. Returns, as characterized in Proposition

2, are completely determined by the beliefs concerning earnings growth and hence inherit the properties of the mixture. The unconditional mixture of the two distributions for the fitted model implies a density which is both negatively skewed (because of the larger proportion of time spent in the high growth state) and has excess-kurtosis. The power of filtering theory becomes evident in agents making a finer distinction of the state of expected earnings growth and hence assigning dynamically evolving mixtures to the two possible distributions. For high beliefs, there is a large chance of a high density and a low chance of a low density creating in sum a negatively skewed density as seen in the top-left panel of Figure 6. Similar logic implies the two densities in the middle and bottom panel.

\section{Calibration and Empirical Implications}

Figure 6 constitutes the heart of the analysis for the two-state earnings growth model and leads to several testable implications for the time-series of option prices. In this section we first present the results of a calibration exercise performed on S\&P 500 real earnings growth and then use the 
parameters and belief process generated to compare the properties of the theoretical and empirically observed option prices. Earnings data are obtained from Standard and Poor's, and the consumption deflator from the Bureau of Economic Analysis.

Maximum likelihood estimates for the parameters are obtained from a regime-switching estimation method as described in Hamilton (1994). ${ }^{11}$ Table 1 contains the parameter values for a 2-state regime switching model from monthly S\&P 500 real earnings growth rates between 1960 and 1998, that are plotted in the top panel of Figure 7. These parameter estimates imply a relatively fast learning process of earnings growth with a double-humped stationary distribution of estimated states, shown in the left panel of Figure 5 . The states are also asymmetric with a larger mass in the higher state of growth. This finding is consistent with the business cycle hypothesis that implies a well known stylized fact, that booms (periods of high growth of stock fundamentals) are of longer duration than recessions (periods of low and often negative growth).

Monthly time-series between 1960:01 and 1998:09 for earnings growth (top panel) and beliefs, $\pi_{t}$, (second panel) are presented in Figure 7. The dark bars indicate NBER dated recessions, periods of declining gross domestic product. As seen in the figure, $\pi_{t}$, the filtered probability of being in the high state, dips during and around each recession of the US economy in the sample, although there have been episodes in three periods, in 1967, in 1986 and in the first half of 1999 when earnings growth slowed even when the overall economy was in an expansionary phase. It should be noted that implicitly we are assuming that investors' information set consists solely of the history of earnings growth. Even though it is unlikely that past earnings are a good proxy for investors' information, the results of this section offer nonetheless a framework to understand certain empirically puzzling phenomena in the options market. The concluding section discusses avenues of future research to refine the empirical methodology. We now turn to the empirical implications of the model.

\footnotetext{
${ }^{11}$ We realize that the estimates of a continuous-time diffusion process for earnings growth based on discrete approximations of the likelihood function may lead to inconsistent estimates for parameters (c.f. for example Lo 1988, AitSahalia 1998). Nonetheless, we argue that for monthly estimation of parameters of the order of magnitude in this paper, the inconsistency will be 'small'. By Example 3 in Lo (1988), the MLE of a discretized log-normal diffusion with constant drift $\theta$ and sampled at intervals of length $h$ converges (as the number of observations increases) to $\hat{\theta}^{h}=\frac{1}{h}\left(e^{\theta h}-1\right)$. For $h=1 / 12$, and for $\theta \in[-.2, .2]$, the inconsistency is of the order $.04 \cdot 1 / 12+O\left(\theta^{2} h^{2}\right) \simeq .003$. Therefore, our results are still informative for the goals of the present paper.
} 


\subsection{Uncertainty and Volatility: ATM Implied Volatility}

The third panel of Figure 7 shows the time series of the CBOE Market Volatility Index and the ATM implied volatility for 1-month options that equates the $C^{\mathrm{U}}\left(z_{t}, K, \pi, T-t\right)$ price as given in (31) with the B-S option price, using the belief process of the calibrated model. The CBOE Market Volatility Index is based on the Black-Scholes implied volatilities of eight near-the-money, nearby, and second nearby OEX options (S\&P 100 Index) and is available on real-time data sources under the ticker VIX. S\&P 100 and 500 returns are highly correlated. To maintain consistency in its composition, the index is constructed so that it represents the implied volatility of a hypothetical at-the-money OEX option with thirty calendar days to expiration. For a complete description of the construction of the index and its composition see Whaley (1993). The figure suggests some association between the two series.

The regressions reported in Table 2 test if the implied volatility series can be explained by the uncertainty about fundamentals using the filtered $\pi_{t}$ process. Line 1 shows that $\pi_{t} \cdot\left(1-\pi_{t}\right)$, which positively affects stock return volatility as seen in (29), has a significant positive coefficient on the time series of implied volatility. Line 2 shows that the coefficient remains significant when we include a lag of implied volatility. The fact that lagged implied volatility is also significant is likely because uncertainty about fundamentals is one of many sources of volatility persistence. For example, there are several studies indicating that trading can also generate persistence in volatility (see, for example, Bollerslev, Chou and Kroner 1992).

To test if asymmetric effects of beliefs on option prices are important we regress separately on $\pi_{t}$ and $\pi_{t}^{2}$. If only $\pi_{t} \cdot\left(1-\pi_{t}\right)$ matters, then we should expect a positive coefficient of $\pi_{t}$ and a negative coefficient of equal size but opposite sign on $\pi_{t}^{2}$. However, if implied volatility is asymmetric about .5, then this restriction will be violated. Implied volatility for the calibrated model (Figure 5 ) is asymmetric about .5, with larger values to the left of .5. However, because most of the observations in the sample are for $\pi_{t}>.5$, and implied volatility is decreasing in $\pi$ in this region, one could get a smaller coefficient (absolute value) on $\pi_{t}$ than on $\pi_{t}^{2}$. As seen in Lines 3 and 4 , this is indeed the case for the sample. Nonetheless, because the standard errors for the coefficients are fairly large, we fail to reject the null of equal coefficients in absolute value.

In Lines 5 and 6 , we test for the coefficients of the theoretical implied volatility calculated using the $\pi_{t}$ series, and the ATM theoretical volatility computed using Proposition 7, with and without a lag of implied volatility. The coefficients are statistically significant, though the $\bar{R}^{2}$ of 
Line 5 is smaller than in line 1 (directly regressing on uncertainty). Finally, in Line 7 , we restrict the regression in Line 5 to the post-crash period, 1988:09 - 1989:09, and obtain an $\bar{R}^{2}$ of almost 17 \%. The improvement in the fit of the theoretical model is not surprising as several authors have noted that the crash of 1987 and the surrounding volatility had little to do with fundamentals.

\subsection{Conditional Skewness of Returns: Ratio of 3\% OTM Put-to-Call Prices}

In this subsection we test if the conditional skewness restriction is indeed decreasing in beliefs as displayed in Figure 6. To efficiently use available option pricing data we construct a test based on the ratio of $3 \%$ OTM Put-to-Call Prices. The ratio is a commonly used measure of market sentiment (c.f. Bates 1999). As evident from Figure 6, the ratio will be larger for higher beliefs as agents have a 'small' probability of a large drop in returns in the event of bad earnings growth realizations. In this and the following subsection, we use option prices and implied volatilities from a sample of daily prices of S\&P 500 call and put options between 1986:04 and 1996:05 traded on the Chicago Board Options Exchange. The underlying index is obtained from the Chicago Mercantile Exchange, and dividend yields are obtained from Standard and Poor's. To conduct the tests we create a monthly time-series of 3\% OTM Put-to-Call Prices interpolated from available prices on the 15th (or closest to 15th) day of each month with approximately 30 days to maturity.

We again run two sets of regressions, which are reported in Table 3, the first directly on the belief process, $\pi_{t}$, (Lines 1 and 2) and the second on the theoretical ratio based on these beliefs but computed using Proposition 7 (Lines 3 and 4). Both variables have statistically significant and positive coefficients with $\bar{R}^{2}$ of about six percent. Including the lag of the dependent variable increases the $\bar{R}^{2}$ to over fifty percent, and the variables remain highly statistically significant. Further intuition for the result can be obtained from the bottom panel of Figure 7 which shows the theoretical correlation between returns and volatility. The theoretical ratio of put-to-call prices is decreasing in this correlation: a negative correlation implies a larger mass for lower returns and hence a higher put price. In passing we note that the empirical ratio fits our model fairly well except in the 1990-1991 period, when in the depths of a recession and negative earnings growth, our model implies a low $\pi_{t}$ and hence a positive correlation between returns and volatility. However as noted by Bates (1999), market participants seemed to have post-crash fears and bid up the ratio to levels far in excess of the theoretical model. Despite the failure of the model's implications in this period, we find explanatory power in the belief process over the 10-year sample. 


\subsection{A Convexity Measure of Implied Volatility - The Implied Volatility of a Butterfly Spread}

In this subsection we test if the conditional kurtosis of returns is increasing in agents' uncertainty, $\pi_{t}\left(1-\pi_{t}\right)$ as displayed in Figure 6 . As seen in the figure, with medium beliefs, the return density is bi-modal with very little mass in its tails; the right panel shows that the implied volatility curve has as 'frown' conditional on these beliefs. Low and high beliefs each have one heavy tail thus generating kurtosis. To efficiently use available option pricing data we construct a test based on a convexity measure of implied volatility $Y_{t}=(\operatorname{Vol}(K / S=97)+\operatorname{Vol}(K / S=103)-2 \operatorname{Vol}(K / S=100))$. Our theoretical model predicts (see Figure 6 , right panels) that $Y_{t}$ is decreasing in $\pi_{t}\left(1-\pi_{t}\right)$.

We once again run two sets of regressions, which are reported in Table 4, the first directly on the uncertainty process, $\pi_{t}\left(1-\pi_{t}\right)$, (Lines 1 and 2) and the second on $Y_{t}^{U}$, the theoretically generated time series using Proposition 7 and using the fitted belief process $\pi_{t}$ (Lines 3 and 4 ). As anticipated, we find statistically significant coefficients, negative for the uncertainty variable and positive for the theoretically generated curvature. The fit of $Y_{t}^{U}$ is slightly better and the variable is more highly statistically significant, but the $\bar{R}^{2}$ from either regression is only around two percent. Including the lag of the dependent variable increases the $\bar{R}^{2}$ to over eight percent, and the variables remain highly statistically significant.

The convexity measure can also be viewed as the implied volatility used in writing a butterfly spread strategy. A butterfly spread strategy involves going long on a call option with a low and a high strike, and short on two call options with the a mid-way exercise price, and is therefore formally identical to the options used in constructing $Y_{t}$. The strategy leads to a profit if the stock price stays close to the mid-way exercise price, but gives rise to a small loss (due to the cost of the options) if there is a significant move in either direction (c.f., for example, Hull and White 1993). Our theoretically generated conditional densities in Figure 6 imply that the options used to replicate the strategy will be priced relatively higher than suggested by the Black-Scholes

formula in periods of high uncertainty, and hence have higher implied volatilities. That $Y_{t}^{U}$ has a significant coefficient in explaining $Y_{t}$ is some evidence that market participants' beliefs have been in line with the calibrated model. 


\subsection{Hedging Implications}

In this subsection we examine some of the hedging implications of the U-Model, in particular focusing the analysis on hedging 'violations' documented empirically in Bakshi et al. (1999). Following Bergman, Grundy and Weiner (1996), these authors noted that one-factor markov option pricing models imply strong restrictions on changes in option prices relative to changes in the underlying: more explicitly, every model in this class must satisfy $0 \leq \Delta_{C} \leq 1$, and $-1 \leq \Delta_{P} \leq 0$ where $\Delta_{C}\left(\Delta_{P}\right)$ is the partial derivative of the call (put) option with respect to the stock price. Taking these implications to high-frequency options data (and ignoring the small time-decay components of option price changes) Bakshi et al. (1999) classify observations satisfying $\Delta C / \Delta S<0$, and $\Delta P / \Delta S>0$ as Type I violations, and $\Delta C / \Delta S>1$, and $\Delta P / \Delta S<-1$ as Type IV violations.

Since B-S falls under the class one-factor markov models, it is unable to explain these violations, which comprise 10-15\% of empirical observations. Bakshi et al. (1999) concluded that in order to improve hedging performance relative to B-S, option pricing models must explicitly have stochastic volatility driven by a second factor. On a similar note, Dumas, Fleming and Whaley (1998) have noted that to improve hedging performance, option pricing models in the Deterministic Volatility Function (DVF) class are unable to improve the hedging performance of option pricing models relative to B-S. In their conclusion they suggest building models in which volatility is a function of past shocks (stock price). Our model, in fact, closely follows this suggestion, because the belief process, which drives volatility, is driven by the the history of shocks to the process $d \tilde{W}$. Even though $d \tilde{W}$ is a $3 \times 1$ vector, the model is easily specialized so that the stock return process is driven by only one Wiener process: Indeed, this happens in the special case where $(i)$ there is no external signal on fundamentals as shown in (2), (ii) the drift of consumption growth is observable and constant, i.e. $\kappa_{1}=\kappa_{2}$ in (3), ${ }^{12}$ and (iii) the covariance between dividend and consumption growth is zero, i.e. $\sigma_{x} \sigma_{c}^{\prime}=0$. Even if there is only one shock (Wiener process), volatility is still driven by beliefs and the covariance between returns and volatility will vary stochastically with beliefs, as elaborated in Proposition 3. This is not possible under one-factor markov or DVF models. Put simply, the stock price in the model is driven by one factor, but is not markov in itself or that one driving factor.

\footnotetext{
${ }^{12}$ Proposition 1 implies that interest rates are constant under this assumption. In addition, for this special case the market price of belief-risk (Corollary 3) is zero. Because return volatility is a function of beliefs, the market price of volatility risk is therefore also zero.
} 
To explicitly address these violations, we study changes in prices of stocks and options on a daily frequency, but must get around the fact that earnings data are received on a monthly frequency and therefore $\Delta \pi$ is not observed daily. We instead construct the 'total' delta with respect to daily changes in the stock price. Partial derivatives with respect to stock prices and beliefs are calculated as shown in Corollary 7. Note that $\Delta C=C_{S} \Delta S+C_{\pi} \Delta \pi=C_{S} \Delta S+C_{\pi} \frac{\partial \pi}{\partial S} \Delta S$. Therefore, replacing $\partial \pi / \partial S$ by the hypothesized relation (10) in Proposition 1 we obtain the total 'delta' with respect to the stock price for the uncertainty model $\Delta_{S}^{U}=C_{S}+C_{\pi} \frac{\pi A+B}{S A}$, and the similar relation for puts where $C_{S}$ and $C_{\pi}$ are as in Corollary 7 .

Total deltas for the model with parameters calibrated to S\&P 500 earnings growth for different levels of moneyness and beliefs are shown in Figure 8. For a given belief, deltas follow the familiar S-shaped pattern for B-S options (c.f. for example Hull 1993). The interesting departures in the figure arise across variations in beliefs. As seen, Type I violations occur for calls that are deep OTM and the agent's belief of the high state is high (larger than .95). Similarly, Type IV violations occur at the diagonally opposite corner, for deep ITM options and low beliefs (smaller than .2). For put options the corners for the two types of violations are switched. The intuition underlying the violations is simple: for $\pi>\hat{\pi}$ the correlation between returns and volatility is negative (Proposition 3) - therefore when the stock price goes up volatility can decline and reduce the option prices leading to a Type I violation for calls. The effect is amplified by the moneyness of the options considered. For $\pi<\hat{\pi}$ the correlation between returns and volatility is positive, and conditional on these beliefs, when returns are positive, volatility increases, and because each effect causes an increase in the option price, a potential Type IV violation is caused. Note that for a model to be consistent with a time-series that has both types of violations, the correlation between returns and volatility must switch signs. We now actually look at the frequency of the violations over a ten-year sample and the ability of the U-model to explain these violations.

Data Description: We consider daily changes in option prices from the 15 th (the day closest to) to the 16th (the following trading day). We consider only maturities of approximately 1-month; we pull on each such day the shortest maturity greater than 30 days. We get options mostly with 34-35 days, although there are a few cases in the range 32-38. The average maturity of options in the sample is 35.3 days. We keep only those options where price quotes are obtained for both days. Overall, we obtain 1021 calls and 1315 puts in the 122 months between 1986:4 1996:5, or about 
8.37 calls and 10.38 puts per month. ${ }^{13}$ We find that the time-series constructed show frequencies of violation similar to those in Bakshi et al. (1999).

In Table 5, we compare the frequency of observed Type I and IV violations in S\&P 500 options in the period between 1986:4 and 1996:5, and the frequency predicted by the U-Model, using the deltas shown in Corollary 7, the belief process shown in Figure 7 and the moneyness of each traded option. As seen for the full sample, the model's predicted level of Type I errors for both calls and puts are quite close to the empirical proportions, but its predictions of Type IV errors fall short. For calls the actual errors are seven times as many as predicted, and for puts the actual proportion is more than twice as large as the predicted proportion. Therefore, for the entire sample, the model is consistent with the prices of calls (puts) and the underlying moving in the opposite (same) direction. It is less able to match the proportion of times that call (put) option prices move in the same (opposite) direction as the stock but by a much greater amount. In a sense, option prices react far more to changes in the underlying than predicted by the calibrated U-model. Figure 8 reveals that the model strongly predicts Type I errors for deep OTM options and Type IV errors for deep ITM options. We next investigate if conditioning on moneyness will help the shortcoming of the model. As seen in the table, by conditioning on moneyness, we are able to increase the predicted proportion of Type IV errors to the empirical proportions for put options. For calls, the model prediction still falls short, although empirical violations happen at higher frequencies, as predicted by the model. However, restricting the moneyness leads to a model overprediction of Type I errors.

In summary, the U-model has some, but not complete, success in explaining violations in changes in call and option prices. The model has very specific implications for regions of violations that can be seen in Figure 8. Empirically, some violations occur for options not in these specified regions, yet conditioning on moneyness increases the empirical violation proportions. Undoubtedly some of the violations will occur for reasons such as market-microstructure effects, idiosyncratic hedging, and dynamic trading strategies, none of which are modeled here. However, the model's accuracy can likely be improved by specifying a richer information set for agents, and a more complex filtering model that will provide greater degrees of freedom for empirical analysis.

\footnotetext{
13 Bakshi et al. (1999) use much finer time-intervals from March 11994 to August 311994 with 3.8 million observations. Nonetheless, they obtain violations for the 1-factor Markov model for all the time-intervals considered, including 1 day.
} 


\section{Conclusions and Extensions}

This paper shows that a simple model in which investors are uncertain about the drift rate of a firm's dividend process is able to shed some light on many of the somewhat puzzling phenomena observed in the option market. Specifically, endogenously determined and belief-dependent stochastic return volatility, stochastic covariance between volatility and returns and a market price for "belief-risk" can account for implied volatility "smirks" and "frowns," as well as their intertemporal instability.

We also obtain semi-closed form solutions for option prices under the assumption of a two-state model using Fourier Transform methods. We compared the option prices implied by our model to Black and Scholes prices and obtained a number of results, such as the following: (a) in the case of symmetric growth rate states, option prices are greater than Black and Scholes' prices when there is high uncertainty; (b) in the case of a persistent high growth state, option prices are highest when investors believe times are bad or uncertain; (c) an increase in risk aversion affects option prices through changes in volatility, dividend yield, interest rate and the market price of belief-risk; (d) across strike prices, implied volatility "smirks" occur in bad or good times, while frowns occur when there is high uncertainty. This latter finding helps in explaining why sudden changes from smiles to frowns occur over time, as documented in the literature.

When calibrated to S\&P 500 real earnings growth for the 1960-1998 sample, the twostate model (consistent with business cycles) implies that changes in market participants' filtered uncertainty about the earnings drift rate helps predict the timing of flips in implied volatility curves between smiles and frowns. The predictive power of filtering theory is further evidenced in the three following time-series tests of the state-return density function from options data: a) ATM implied volatility (persistence), b) Ratio of 3\% OTM put-to-call prices (skewness), and (c) Implied Volatility of a $3 \%$ butterfly spread (kurtosis). A special case of the model where the stock process is driven by a single Wiener process is also consistent with empirically observed hedging 'violations' (i.e. overreactions and opposite (same) sign changes in the underlying and call (put) prices) that are not compatible with one-factor markov and deterministic volatility function models, because the dividend yield and return volatility are driven by the entire path of the Wiener process summarized in the investor's posterior probability distribution.

An important omission in the calibration exercises conducted in Section 5 is the appropriate modeling of the relationship between the agent's and econometrician's information sets. The two simple exercises assume that they are equal and solely consist of the history of earnings growth. 
Our theoretical framework in Section 1 is consistent with agents' receiving unbiased signals on drift rates, leading to one possible extension for the calibration. ${ }^{14}$ More generally, because it is going to be hard to completely specify agents' information sets, an avenue for future research would be to back out the uncertainty from option prices.

\footnotetext{
${ }^{14}$ Our study of this limited information set suggests a fast learning asymmetric growth model. More precise signals can only lead to faster learning than calibrated, but will lead to the stationary density of the same but possibly more concentrated shape. Therefore, the qualitative predictions on implied volatility changes will be preserved.
} 


\section{Appendix 1.}

Proof of Proposition 1: Rewrite (9) as

$$
S(t)=x(t) E_{t}\left[\int_{t}^{\infty} \frac{m(s) x(s)}{m(t) x(t)} d s\right]=x(t) \sum_{i=1}^{n} E_{t}\left[\int_{t}^{\infty} \frac{m(s) x(s)}{m(t) x(t)} d s \mid \nu(t)=\nu_{i}\right] \pi_{i}(t)
$$

Define $M(s)=m(s) x(s)$ to find

$$
\begin{aligned}
d M= & x d m+m d x+d m d x \\
= & M\left[\left(-\phi-\gamma \kappa(t)+\frac{1}{2} \gamma(\gamma+1) \sigma_{c} \sigma_{c}^{\prime}\right) d t-\gamma \sigma_{c} d W\right] \\
& +M\left[\theta(t) d t+\sigma_{x} d W\right]-M \gamma \sigma_{c} \sigma_{x} d t \\
= & M\left[-\phi-\gamma \kappa(t)+\theta(t)+\frac{1}{2} \gamma(\gamma+1) \sigma_{c} \sigma_{c}^{\prime}-\gamma \sigma_{c} \sigma_{x}\right] d t+M\left(\iota_{x}^{\prime}-\gamma \iota_{c}^{\prime}\right) \Sigma d W
\end{aligned}
$$

For convenience, define $\widehat{\theta}(t)=\phi+\gamma \kappa(t)-\theta(t)-\frac{1}{2} \gamma(\gamma+1) \sigma_{c} \sigma_{c}^{\prime}+\gamma \sigma_{c} \sigma_{x}$ so that

$$
\frac{d M}{M}=-\widehat{\theta}(t) d t+\left(\iota_{x}-\gamma \iota_{c}\right)^{\prime} \Sigma d W
$$

Hence

$$
\frac{M(s)}{M(t)}=\exp \left(-\int_{t}^{s}\left(\widehat{\theta}(u)-\frac{1}{2}\left(\iota_{x}-\gamma \iota_{c}\right)^{\prime} \Sigma \Sigma^{\prime}\left(\iota_{x}-\gamma \iota_{c}\right)\right) d u+\left(\iota_{x}-\gamma \iota_{c}\right)^{\prime} \Sigma(W(s)-W(t))\right)
$$

By substituting into (35), we obtain the same set up as in the proof of proposition 3.1 in Veronesi (1999a). Using the same methodology, we find that for all $i=1, \ldots, n$

$$
E_{t}\left[\int_{t}^{\infty} \frac{M(s)}{M(t)} d s \mid \nu(t)=\nu_{i}\right]=C_{i}
$$

for constants $C=\left(C_{1}, \ldots, C_{n}\right)$ defined as follows: let

$$
A=-\Lambda+\operatorname{diag}(\widehat{\theta})=-\Lambda+\operatorname{diag}(\gamma \kappa-\theta)+\left(\phi-\frac{1}{2} \gamma(\gamma+1) \sigma_{c} \sigma_{c}^{\prime}+\gamma \sigma_{c} \sigma_{x}^{\prime}\right) I_{n}
$$

then

$$
C=A^{-1} 1_{n}
$$

Notice finally that if the condition of proposition 1 (a) in the text is satisfied, then $\hat{\theta}(t)$ is always positive. From (36), this ensures that $M(t)>0$ for all $t>0$. Hence, from (37) we also have that $C_{i}>0$ for all $i$ 's.

The proof of (b) is as in Veronesi (1999a) and the proof of (c) is as in Yared (1999) and Veronesi and Yared (1999) 
Proof of Corollary 1: From (6) we can write $\Sigma d W=-(v(t)-\bar{v}) d t+\left(\Sigma \Sigma^{\prime}\right)^{\frac{1}{2}} d \widetilde{W}$ and hence

$$
\frac{d y}{y}=v(t) d t+\Sigma d W=\bar{v} d t+\left(\Sigma \Sigma^{\prime}\right)^{\frac{1}{2}} d \widetilde{W}
$$

Proof of Proposition 2: Notice that the following covariances hold:

$$
\begin{aligned}
d \pi_{i} d \pi_{j}^{\prime} & =\pi_{i} \pi_{j}\left(\nu_{i}-\bar{\nu}\right)^{\prime}\left(\Sigma \Sigma^{\prime}\right)^{-1}\left(\nu_{j}-\bar{\nu}\right) d t \\
d x d \pi_{i}^{\prime} & =x \iota_{x}^{\prime}\left(\Sigma \Sigma^{\prime}\right)^{\frac{1}{2}}\left(\Sigma \Sigma^{\prime}\right)^{-\frac{1}{2}}\left(\nu_{i}-\bar{\nu}\right) \pi_{i}=\left(\theta_{i}-\bar{\theta}\right) \pi_{i} x \\
d e d \pi_{i}^{\prime} & =e \iota_{e}^{\prime}\left(\Sigma \Sigma^{\prime}\right)^{\frac{1}{2}}\left(\Sigma \Sigma^{\prime}\right)^{-\frac{1}{2}}\left(\nu_{i}-\bar{\nu}\right) \pi_{i}=\left(\theta_{i}-\bar{\theta}\right) \pi_{i} e \\
d c d \pi_{i}^{\prime} & =c \iota_{c}^{\prime}\left(\Sigma \Sigma^{\prime}\right)^{\frac{1}{2}}\left(\Sigma \Sigma^{\prime}\right)^{-\frac{1}{2}}\left(\nu_{i}-\bar{\nu}\right) \pi_{i}=\left(\kappa_{i}-\bar{\kappa}\right) \pi_{i} c
\end{aligned}
$$

Hence, from Ito's lemma:

$$
\begin{aligned}
d S & =\sum_{i=1}^{n} C_{i} \pi_{i} d x+x \sum_{i=1}^{n} C_{i} d \pi_{i}+\sum_{i=1}^{n} C_{i} d x d \pi_{i}^{\prime} \\
& =S\left(\bar{\theta} d t+\iota_{x}^{\prime}\left(\Sigma \Sigma^{\prime}\right)^{\frac{1}{2}} d \widetilde{W}+\frac{\sum_{i=1}^{n} C_{i}[\pi \Lambda]_{i}}{\sum_{i=1}^{n} C_{i} \pi_{i}} d t+\frac{\sum_{i=1}^{n} C_{i} \pi_{i}\left(\nu_{i}-\bar{\nu}\right)^{\prime}\left(\Sigma \Sigma^{\prime}\right)^{-\frac{1}{2}} d \widetilde{W}}{\sum_{i=1}^{n} C_{i} \pi_{i}}+\frac{\sum_{i=1}^{n} C_{i} \pi_{i}\left(\theta_{i}-\bar{\theta}\right)}{\sum_{i=1}^{n} C_{i} \pi_{i}} d t\right) \\
& =S\left(\bar{\theta}+\frac{\pi \Lambda C}{\sum_{i=1}^{n} C_{i} \pi_{i}}+\frac{\sum_{i=1}^{n} C_{i} \pi_{i}\left(\theta_{i}-\bar{\theta}\right)}{\sum_{i=1}^{n} C_{i} \pi_{i}}\right) d t+S\left(\iota x\left(\Sigma \Sigma^{\prime}\right)^{\frac{1}{2}}+\frac{\sum_{i=1}^{n} C_{i} \pi_{i}\left(\nu_{i}-\bar{\nu}\right)^{\prime}\left(\Sigma \Sigma^{\prime}\right)^{-\frac{1}{2}}}{\sum_{i=1}^{n} C_{i} \pi_{i}}\right) d \widetilde{W}
\end{aligned}
$$

Hence, $\sigma_{S}$ is as defined in proposition 2. To get the result about $\mu_{S}$ notice that from (38):

$$
\pi \Lambda C=\sum_{i=1}^{n} \pi_{i} C_{i}\left(\gamma \kappa_{i}-\theta_{i}\right)+\left(\phi-\frac{1}{2} \gamma(\gamma+1) \sigma_{c} \sigma_{c}^{\prime}+\gamma \sigma_{c} \sigma_{x}^{\prime}\right) \sum_{i=1}^{n} \pi_{i} C_{i}-1
$$

Hence the expected capital gain return can be rewritten as:

$$
\begin{aligned}
E_{t}\left(\frac{d S}{S}\right) & =\bar{\theta}+\frac{\sum_{i=1}^{n} \pi_{i} C_{i}\left(\gamma \kappa_{i}-\theta_{i}\right)-1}{\sum_{i=1}^{n} C_{i} \pi_{i}}+\left(\phi-\frac{1}{2} \gamma(\gamma+1) \sigma_{c} \sigma_{c}^{\prime}+\gamma \sigma_{c} \sigma_{x}^{\prime}\right)+\frac{\sum_{i=1}^{n} C_{i} \pi_{i}\left(\theta_{i}-\bar{\theta}\right)}{\sum_{i=1}^{n} C_{i} \pi_{i}} \\
& =\gamma \frac{\sum_{i=1}^{n} \pi_{i} C_{i} \kappa_{i}}{\sum_{i=1}^{n} C_{i} \pi_{i}}-\frac{1}{\sum_{i=1}^{n} C_{i} \pi_{i}}+\phi-\frac{1}{2} \gamma(\gamma+1) \sigma_{c} \sigma_{c}^{\prime}+\gamma \sigma_{c} \sigma_{x}^{\prime} \\
& =\gamma \frac{\sum_{i=1}^{n} \pi_{i} C_{i} \kappa_{i}}{\sum_{i=1}^{n} C_{i} \pi_{i}}-\frac{x}{S}+r-\gamma \bar{\kappa}+\gamma \sigma_{c} \sigma_{x}^{\prime}
\end{aligned}
$$

Finally, to show equation (12) we can see that since

$$
\begin{aligned}
\operatorname{Cov}\left(\frac{d S}{S}, \frac{d c}{c}\right) & =\sigma_{S} \sigma_{c}^{\prime}=\left(\iota_{x}^{\prime}\left(\Sigma \Sigma^{\prime}\right)^{\frac{1}{2}}+\frac{\sum_{i=1}^{n} C_{i} \pi_{i}\left(\nu_{i}-\bar{\nu}\right)^{\prime}\left(\Sigma \Sigma^{\prime}\right)^{-\frac{1}{2}}}{\sum_{i=1}^{n} C_{i} \pi_{i}}\right)\left(\Sigma \Sigma^{\prime}\right)^{\frac{1}{2}} \iota_{c} \\
& =\iota_{x}^{\prime} \Sigma \Sigma^{\prime} \iota_{c}+\frac{\sum_{i=1}^{n} C_{i} \pi_{i}\left(\kappa_{i}-\bar{\kappa}\right)}{\sum_{i=1}^{n} C_{i} \pi_{i}}
\end{aligned}
$$




$$
=\sigma_{c} \sigma_{x}^{\prime}+\frac{\sum_{i=1}^{n} C_{i} \pi_{i}\left(\kappa_{i}-\bar{\kappa}\right)}{\sum_{i=1}^{n} C_{i} \pi_{i}}
$$

The result follows immediately.

Proof of Proposition 3: (a) From the definition of $\pi^{*}$ and $b$ we can rewrite: $\sigma_{S}=\iota_{x}\left(\Sigma \Sigma^{\prime}\right)^{\frac{1}{2}}+\left(\bar{\theta}^{*}-\bar{\theta}\right) b$

We then have immediately $V=\sigma_{x}^{2}+2\left(\bar{\theta}^{*}-\bar{\theta}\right)+\left(\bar{\theta}^{*}-\bar{\theta}\right)^{2} b b^{\prime}$

(b) Notice first

$$
\begin{aligned}
\frac{\partial\left(\bar{\theta}^{*}-\bar{\theta}\right)}{\partial \pi_{i}} & =\frac{\partial\left(\frac{\sum_{j} \pi_{j} C_{j} \theta_{j}}{\sum_{j} \pi_{j} C_{j}}-\sum_{j} \pi_{j} \theta_{j}\right)}{\partial \pi_{i}}=\frac{C_{i} \theta_{i}\left(\sum_{j} \pi_{j} C_{j}\right)-C_{i}\left(\sum_{j} \pi_{j} C_{j} \theta_{j}\right)}{\left(\sum_{j} \pi_{j} C_{j}\right)^{2}}-\theta_{i} \\
& =\frac{C_{i}\left(\theta_{i}-\bar{\theta}^{*}\right)}{\left(\sum_{j} \pi_{j} C_{j}\right)}-\theta_{i}
\end{aligned}
$$

Hence,

$$
\begin{aligned}
\frac{\partial V}{\partial \pi_{i}} & =2 \frac{\partial\left(\bar{\theta}^{*}-\bar{\theta}\right)}{\partial \pi_{i}}+2\left(\bar{\theta}^{*}-\bar{\theta}\right) b b^{\prime} \frac{\partial\left(\bar{\theta}^{*}-\bar{\theta}\right)}{\partial \pi_{i}}=2 \frac{\partial\left(\bar{\theta}^{*}-\bar{\theta}\right)}{\partial \pi_{i}}\left(1+\left(\bar{\theta}^{*}-\bar{\theta}\right) b b^{\prime}\right) \\
& =2\left(\frac{C_{i}\left(\theta_{i}-\bar{\theta}^{*}\right)}{\left(\sum_{j} \pi_{j} C_{j}\right)}-\theta_{i}\right)\left(1+\left(\bar{\theta}^{*}-\bar{\theta}\right) b b^{\prime}\right)
\end{aligned}
$$

and

$$
\begin{aligned}
\frac{\partial^{2} V}{\partial \pi_{i} \partial \pi_{j}}= & 2\left(\frac{-\left(\frac{\partial \bar{\theta}^{*}}{\partial \pi_{j}}\right) C_{i}\left(\sum_{k} \pi_{k} C_{k}\right)-C_{i} C_{j}\left(\theta_{i}-\bar{\theta}^{*}\right)}{\left(\sum_{k} \pi_{k} C_{k}\right)^{2}}\right)\left(1+\left(\bar{\theta}^{*}-\bar{\theta}\right) b b^{\prime}\right) \\
& +2\left(\frac{C_{i}\left(\theta_{i}-\bar{\theta}^{*}\right)}{\left(\sum_{k} \pi_{k} C_{k}\right)}-\theta_{i}\right)\left(\frac{\partial \bar{\theta}^{*}}{\partial \pi_{j}}-\theta_{j}\right) b b^{\prime} \\
= & -2\left(\frac{C_{j} C_{i}\left(\theta_{j}-\bar{\theta}^{*}\right)+C_{i} C_{j}\left(\theta_{i}-\bar{\theta}^{*}\right)}{\left(\sum_{k} \pi_{k} C_{k}\right)^{2}}\right)\left(1+\left(\bar{\theta}^{*}-\bar{\theta}\right) b b^{\prime}\right) \\
& +2\left(\frac{C_{i}\left(\theta_{i}-\bar{\theta}^{*}\right)}{\left(\sum_{k} \pi_{k} C_{k}\right)}-\theta_{i}\right)\left(\frac{C_{j}\left(\theta_{j}-\bar{\theta}^{*}\right)}{\left(\sum_{k} \pi_{k} C_{k j}\right)}-\theta_{j}\right) b b^{\prime}
\end{aligned}
$$

We can then write:

$$
\begin{aligned}
d V & =\sum_{i=1}^{N} \frac{\partial V}{\partial \pi_{i}} d \pi_{i}+\frac{1}{2} \sum_{i} \sum_{j} \frac{\partial^{2} V}{\partial \pi_{i} \partial \pi_{j}} d \pi_{i} d \pi_{j} \\
& =\left(\sum_{i=1}^{N} \frac{\partial V}{\partial \pi_{i}} \mu_{i}+\frac{1}{2} \sum_{i} \sum_{j} \frac{\partial^{2} V}{\partial \pi_{i} \partial \pi_{j}} \sigma_{i} \sigma_{j}\right) d t+\sum_{i=1}^{N} \frac{\partial V}{\partial \pi_{i}} \sigma_{i} d \widetilde{W}
\end{aligned}
$$




$$
=\mu_{V} d t+\sigma_{V} d \widetilde{W}
$$

where we can rewrite $\sigma_{i}=\pi_{i}\left(\theta_{i}-\bar{\theta}\right) b$. We then have

$$
\mu_{V}=\sum_{i=1}^{N} \frac{\partial V}{\partial \pi_{i}} \mu_{i}+\frac{1}{2} \sum_{i} \sum_{j} \frac{\partial^{2} V}{\partial \pi_{i} \partial \pi_{j}} \sigma_{i} \sigma_{j}
$$

Consider the two terms separately

$$
\begin{aligned}
\sum_{i=1}^{N} \frac{\partial V}{\partial \pi_{i}} \mu_{i} & =\sum_{i=1}^{N} 2\left(\frac{C_{i}\left(\theta_{i}-\bar{\theta}^{*}\right)}{\left(\sum_{k} \pi_{k} C_{k}\right)}-\theta_{i}\right)\left(1+\left(\bar{\theta}^{*}-\bar{\theta}\right) b b^{\prime}\right)\left(\sum_{j=1}^{n} \pi_{j} \lambda_{j i}\right) \\
& =2 \sum_{i=1}^{N}\left(\frac{[\pi \Lambda]_{i} C_{i}\left(\theta_{i}-\bar{\theta}^{*}\right)}{\left(\sum_{k} \pi_{k} C_{k}\right)}-\sum_{j} \pi_{j} \lambda_{j i} \theta_{i}\right)\left(1+\left(\bar{\theta}^{*}-\bar{\theta}\right) b b^{\prime}\right)
\end{aligned}
$$

Lengthy algebra also shows:

$$
\frac{1}{2} \sum_{i} \sum_{j} \frac{\partial^{2} V}{\partial \pi_{i} \partial \pi_{j}} \sigma_{i} \sigma_{j}=\left(\sigma_{\theta}^{* 2}-\sigma_{\theta}^{2}\right)^{2}\left(b b^{\prime}\right)^{2}-2 \sigma_{\theta}^{* 2}\left(\bar{\theta}^{*}-\bar{\theta}\right) b b^{\prime}\left(1+\left(\bar{\theta}^{*}-\bar{\theta}\right) b b^{\prime}\right)
$$

where the only tricky part is to make use of the definition of $\pi_{i}^{*}$ whenever possible and notice also that

$$
\sum_{j}\left(\pi_{j}^{*}\left(\theta_{j}-\bar{\theta}^{*}\right)\left(\theta_{j}-\bar{\theta}\right)\right)=\sum_{j} \pi_{j}^{*} \theta_{j}^{2}-\left(\bar{\theta}^{*}\right)^{2}=\sigma_{\theta}^{* 2}
$$

Overall:

$$
\begin{aligned}
\mu_{V}= & 2 \sum_{i=1}^{N}\left(\frac{[\pi \Lambda]_{i} C_{i}\left(\theta_{i}-\bar{\theta}^{*}\right)}{\left(\sum_{k} \pi_{k} C_{k}\right)}-\sum_{j} \pi_{j} \lambda_{j i} \theta_{i}\right)\left(1+\left(\bar{\theta}^{*}-\bar{\theta}\right) b b^{\prime}\right) \\
& +\left(\sigma_{\theta}^{* 2}-\sigma_{\theta}^{2}\right)^{2}\left(b b^{\prime}\right)^{2}-2 \sigma_{\theta}^{* 2}\left(\bar{\theta}^{*}-\bar{\theta}\right) b b^{\prime}\left(1+\left(\bar{\theta}^{*}-\bar{\theta}\right) b b^{\prime}\right) \\
= & 2\left(E_{t}\left(d \bar{\theta}^{*}\right)-E_{t}(d \bar{\theta})\right)\left(1+\left(\bar{\theta}^{*}-\bar{\theta}\right) b b^{\prime}\right)+\left(\sigma_{\theta}^{* 2}-\sigma_{\theta}^{2}\right)^{2}\left(b b^{\prime}\right)^{2}
\end{aligned}
$$

where by direct calculation one can show that

$$
E_{t}\left(d \bar{\theta}^{*}\right)=\left(\frac{\sum_{i}[\pi \Lambda]_{i} C_{i}\left(\theta_{i}-\bar{\theta}^{*}\right)}{\left(\sum_{j} \pi_{j} C_{j}\right)}-\sigma_{\theta}^{* 2}\left(\bar{\theta}^{*}-\bar{\theta}\right) b b^{\prime}\right)
$$

Finally,

$$
\sigma_{V}=\sum_{i=1}^{N} \frac{\partial V}{\partial \pi_{i}} \sigma_{i}=\sum_{i=1}^{N}\left(\frac{C_{i}\left(\theta_{i}-\bar{\theta}^{*}\right)}{\left(\sum_{j} \pi_{j} C_{j}\right)}-\theta_{i}\right) \pi_{i}\left(\theta_{i}-\bar{\theta}\right)\left(1+\left(\bar{\theta}^{*}-\bar{\theta}\right) b b^{\prime}\right) 2 b
$$




$$
=\left(\sigma_{\theta}^{* 2}-\sigma_{\theta}^{2}\right)\left(1+\left(\bar{\theta}^{*}-\bar{\theta}\right) b b^{\prime}\right) 2 b
$$

From here, we also obtain

$$
\operatorname{Cov}(d V, d R)=\sigma_{V} \sigma_{S}^{\prime}=2\left(\sigma_{\theta}^{* 2}-\sigma_{\theta}^{2}\right)\left(1+\left(\bar{\theta}^{*}-\bar{\theta}\right) b b^{\prime}\right)^{2}
$$

This concludes the proof.

Proof of Corollary 2: Let $\pi=\pi_{2}$. In the two-state case with $\kappa_{1}=\kappa_{2}$ we have

$$
\bar{\theta}^{*}-\bar{\theta}=\frac{\pi(1-\pi)\left(C_{2}-C_{1}\right)\left(\theta_{2}-\theta_{1}\right)}{\pi\left(C_{2}-C_{1}\right)+C_{2}}
$$

Hence, the volatility function $V$ in equation (17) can be written as $V=\sigma_{x} \sigma_{x}^{\prime}+K_{1} X+K_{2} X^{2}$ where $X=g(\pi)=\frac{\pi(1-\pi)}{\pi\left(C_{2}-C_{1}\right)+C_{1}}, K_{1}=2\left(C_{2}-C_{1}\right)\left(\theta_{2}-\theta_{1}\right)$ and $K_{2}=\left(C_{2}-C_{1}\right)^{2}\left(\theta_{2}-\theta_{1}\right)^{2} b b^{\prime}$. Since $0<\pi<1$, we have that the domain of $X$ is the interval $[0, \bar{X}]$ for some $\bar{X}$. Since both $K_{1}$ and $K_{2}$ are positive, $V$ is increasing on $[0, \bar{X}]$ and hence it has a unique maximum at $\bar{X}$. In addition, we see that the function $X=g(\pi)$ has negative second derivative, implying that it has a unique maximum as well. Hence, we can define $\hat{\pi}$ such that $\bar{X}=\max _{\pi} g(\pi)=g(\hat{\pi})$. In conclusion, the function $V$ has a unique maximum at $\hat{\pi}$. Since the function is continuous, we must have $V_{\pi}<0$ if and only if $\pi>\hat{\pi}$. Finally, using Ito's Lemma we can always write

$$
\begin{aligned}
d V & =\mu_{V} d t+V_{\pi} d \pi \\
& =\tilde{\mu}_{V} d t+V_{\pi} \pi(1-\pi)\left(\theta_{2}-\theta_{1}\right) b d \tilde{W}
\end{aligned}
$$

for some drift term $\tilde{\mu}_{V}$. Hence

$$
\operatorname{Cov}\left(d V, \frac{d S}{S}\right)=V_{\pi} \pi(1-\pi)\left(\theta_{2}-\theta_{1}\right)\left(b \sigma_{S}^{\prime}\right)
$$

It follows that the sign of $\operatorname{Cov}\left(d V, \frac{d S}{S}\right)$ is the same as the sign of $V_{\pi}$, because all the other terms are positive.

Proof of Proposition 4: Since we have 3 Brownian motions, we need 4 traded securities in order to obtain a riskless portfolio. ${ }^{15}$ One of course is $S$, and let $f_{i}$ for $i=1,2,3$ be three other traded securities, each following the process

$$
\frac{d f_{i}}{f_{i}}=\mu_{f i} d t+\sigma_{f i} d \widetilde{W}
$$

\footnotetext{
${ }^{15}$ The following derivation is standard. See e.g. Hull(1993).
} 
where

$$
\begin{aligned}
f_{i} \mu_{f_{i}}= & \frac{\partial f_{i}}{\partial t}+\frac{\partial f_{i}}{\partial S}\left(\mu_{S}-\delta\right) S+\sum_{j=1}^{n-1} \frac{\partial f_{i}}{\partial \pi_{j}} \mu_{j}+\frac{1}{2} \frac{\partial^{2} f_{i}}{\partial S^{2}} \sigma_{S} \sigma_{S}^{\prime} S^{2}+\sum_{j=1}^{n-1} \frac{\partial^{2} f_{i}}{\partial S \partial \pi_{j}} S \sigma_{S} \sigma_{j}^{\prime} \\
& +\frac{1}{2} \sum_{k=1}^{n-1} \sum_{j=1}^{n-1} \frac{\partial f_{i}}{\partial \pi_{k} \partial \pi_{j}} \sigma_{k} \sigma_{j}^{\prime} \\
f_{i} \sigma_{f i, \ell}= & \frac{\partial f_{i}}{\partial S} S \sigma_{S, \ell}+\sum_{j=1}^{n-1} \frac{\partial f_{i}}{\partial \pi_{j}} \sigma_{j, \ell}
\end{aligned}
$$

for $\ell=1,2,3$, where $\sigma_{f i, \ell}$ is the $\ell$-th component in the volatility vector $\sigma_{f i}$. Hence, consider the portfolio

$$
H=N_{0} S+\sum_{i=1}^{3} N_{i} f_{i}
$$

where $N_{i}$ 's are the amount of each security in the portfolio. From Ito's lemma we can make $d H$ riskless by choosing $N_{i}$ 's so that for all $\ell=1,2,3$

$$
N_{0} S \sigma_{S, \ell}+\sum_{i=1}^{3} N_{i} f_{i} \sigma_{f i, \ell}=0
$$

If $H$ is riskless, we then have

$$
N_{0} S \mu_{S}+\sum_{i=1}^{3} N_{i} f_{i} \mu_{f i}=r\left(N_{0} S+\sum_{i=1}^{3} N_{i} f_{i}\right)
$$

or

$$
N_{0} S\left(\mu_{S}-r\right)+\sum_{i=1}^{3} N_{i} f_{i}\left(\mu_{f i}-r\right)=0
$$

The last equation and (40) imply that there must be $\psi_{1}, \psi_{2}, \psi_{3}$ such that

$$
\begin{aligned}
& \mu_{S}-r=\sum_{\ell=1}^{3} \psi_{\ell} \sigma_{S, \ell} \\
& \mu_{f i}-r=\sum_{\ell=1}^{3} \psi_{\ell} \sigma_{f i, \ell}
\end{aligned}
$$

for all $i$ 's. Using the last equality and the definitions of $\mu_{f i}$ and $\sigma_{f i}$, and dropping he subscript $i$, we have that every derivative security must satisfy the following PDE

$$
\frac{\partial f}{\partial t}+\frac{\partial f}{\partial S}\left(\mu_{S}-\delta\right) S+\sum_{j=1}^{n-1} \frac{\partial f}{\partial \pi_{j}} \mu_{j}+\frac{1}{2} \frac{\partial^{2} f}{\partial S^{2}} \sigma_{S} \sigma_{S}^{\prime} S^{2}+\sum_{j=1}^{n-1} \frac{\partial^{2} f}{\partial S \partial \pi_{j}} S \sigma_{S} \sigma_{i}^{\prime}+\frac{1}{2} \sum_{k=1}^{n-1} \sum_{j=1}^{n-1} \frac{\partial f}{\partial \pi_{k} \partial \pi_{j}} \sigma_{k} \sigma_{j}^{\prime}
$$




$$
=r f+\sum_{\ell=1}^{3} \psi_{\ell}\left(\frac{\partial f}{\partial S} S \sigma_{S, \ell}+\sum_{j=1}^{n-1} \frac{\partial f}{\partial \pi_{j}} \sigma_{j, \ell}\right)
$$

or

$$
\begin{aligned}
r f= & \frac{\partial f}{\partial t}+\frac{\partial f}{\partial S} S(r-\delta)+\sum_{j=1}^{n-1} \frac{\partial f}{\partial \pi_{j}}\left(\mu_{j}-\sum_{\ell=1}^{3} \psi_{\ell} \sigma_{j, \ell}\right)+\frac{1}{2} \frac{\partial^{2} f}{\partial S^{2}} S^{2} \sigma_{S} \sigma_{S}^{\prime} \\
& +\sum_{j=1}^{n-1} \frac{\partial^{2} f}{\partial S \partial \pi_{j}} S \sigma_{S} \sigma_{i}^{\prime}+\frac{1}{2} \sum_{k=1}^{n-1} \sum_{j=1}^{n-1} \frac{\partial f}{\partial \pi_{k} \partial \pi_{j}} \sigma_{k} \sigma_{j}^{\prime}
\end{aligned}
$$

Finally, comparing (41) to (12) and noticing that $\operatorname{Cov}\left(\frac{d c}{c}, \frac{d S}{S}\right)=\iota_{c}\left(\Sigma \Sigma^{\prime}\right)^{\frac{1}{2}} \sigma_{S}^{\prime}$ we obtain that the vector $\psi=\left(\psi_{1}, \psi_{2}, \psi_{3}\right)=\gamma \iota_{c}\left(\Sigma \Sigma^{\prime}\right)^{\frac{1}{2}}$. Hence the market price of $\pi_{j}$ risk is

$$
\sum_{\ell=1}^{3} \psi_{\ell} \sigma_{j, \ell}=\gamma \iota_{c}\left(\Sigma \Sigma^{\prime}\right)^{\frac{1}{2}} \sigma_{j}^{\prime}=\gamma \pi_{j}\left(\kappa_{j}-\bar{\kappa}\right)=\gamma \operatorname{Cov}\left(\frac{d c}{c}, d \pi_{j}\right)
$$

Proof of Lemma 2: Let the distribution $\pi$ be given, so that we can treat $\sum_{i} \pi_{i} C_{i}$ as a constant. If $C_{i}$ are increasing in $i$, we also have $\frac{C_{i}}{\sum_{i} \pi_{i} C_{i}}$ are monotonically increasing. Notice also that we must have

$$
\frac{C_{0}}{\sum_{i} \pi_{i} C_{i}}<1<\frac{C_{n}}{\sum_{i} \pi_{i} C_{i}}
$$

Hence, by definition of $\pi_{i}^{*}$ there must exist a $k$ such that we have that $\pi_{i}^{*}<\pi_{i}$ for $i \leq k$ and $\pi_{i}^{*}>\pi_{i}$ for $i>k$. Since $\theta_{1}<\ldots<\theta_{n}$, their average computed using $\pi^{*}$ must be greater than their average computed using $\pi$. Hence $\bar{\theta}^{*}>\bar{\theta}$.

Proof of Proposition 5: Part (a) is immediate from Lemma 2.

(b) Let $\widehat{\pi}$ be a mean preserving spread of the distribution $\pi$. If $f_{i}=C_{i} \times\left(\theta_{i}-\theta_{n}\right)$ is convex then a mean preserving spread increases both the quantity $\sum_{i=1}^{n} f_{i} \pi_{i}$ and the quantity $\sum_{i=1}^{n} C_{i} \pi_{i}$, that is

$$
\sum_{i=1}^{n} f_{i} \pi_{i}<\sum_{i=1}^{n} f_{i} \widehat{\pi}_{i}<0<\sum_{i=1}^{n} C_{i} \pi_{i}<\sum_{i=1}^{n} C_{i} \widehat{\pi}_{i}
$$

We then have

$$
\begin{aligned}
\frac{\sum_{i=1}^{n} C_{i} \widehat{\pi}_{i}\left(\theta_{i}-\bar{\theta}\right)}{\sum_{i=1}^{n} C_{i} \widehat{\pi}_{i}} & =\frac{\sum_{i=1}^{n} C_{i} \widehat{\pi}_{i} \theta_{i}}{\sum_{i=1}^{n} C_{i} \widehat{\pi}_{i}}-\sum_{i=1}^{n} \widehat{\pi}_{i} \theta_{i}=\frac{\sum_{i=1}^{n} C_{i} \widehat{\pi}_{i}\left(\theta_{i}-\theta_{n}\right)}{\sum_{i=1}^{n} C_{i} \widehat{\pi}_{i}}+\theta_{n}-\sum_{i=1}^{n} \widehat{\pi}_{i} \theta_{i} \\
& =\frac{\sum_{i=1}^{n} \widehat{\pi}_{i} f_{i}}{\sum_{i=1}^{n} C_{i} \widehat{\pi}_{i}}+\theta_{n}-\sum_{i=1}^{n} \widehat{\pi}_{i} \theta_{i} \\
& >\frac{\sum_{i=1}^{n} \pi_{i} f_{i}}{\sum_{i=1}^{n} C_{i} \pi_{i}}+\theta_{n}-\sum_{i=1}^{n} \pi_{i} \theta_{i}=\frac{\sum_{i=1}^{n} C_{i} \pi_{i}\left(\theta_{i}-\bar{\theta}\right)}{\sum_{i=1}^{n} C_{i} \pi_{i}}
\end{aligned}
$$


Hence, a mean preserving spread increases the quantity

$$
\sigma_{S} \sigma_{S}^{\prime}=\sigma_{x} \sigma_{x}^{\prime}+2 \frac{\sum_{i=1}^{n} C_{i} \pi_{i}\left(\theta_{i}-\bar{\theta}\right)}{\sum_{i=1}^{n} C_{i} \pi_{i}}+\left(\frac{\sum_{i=1}^{n} C_{i} \pi_{i}\left(\theta_{i}-\bar{\theta}\right)}{\sum_{i=1}^{n} C_{i} \pi_{i}}\right)^{2} b b^{\prime}
$$

(c) Since $b b^{\prime}=(1,1,0) \times\left(\Sigma \Sigma^{\prime}\right)^{-1} \times(1,1,0)^{\prime}=\left(v_{11}+v_{22}\right)$ where $v_{i j}$ is the $i j$-the element of $\left(\Sigma \Sigma^{\prime}\right)^{-1}$, we see immediately that decreasing $\sigma_{2 e}$ implies increasing $v_{22}=1 / \sigma_{2 e}^{2}$. The result follows immediately.

Proof of Proposition 6: In cases (I) and (II) we can rewrite

$$
C_{i}=\frac{C_{1} \times\left(G(\gamma)-\theta_{1}\right)}{G(\gamma)-\theta_{i}}
$$

From the definition of the dividend yield and the application of the chain rule, it is immediate to show that the dividend yield is increasing in $\gamma$. We now show that

$$
V=\frac{\sum_{i=1}^{n} C_{i} \pi_{i} \theta_{i}}{\sum_{i=1}^{n} C_{i} \pi_{i}}
$$

is decreasing in $\gamma$. The result immediately follows. Specifically, because

$$
\frac{\partial V}{\partial \gamma}=\frac{\partial V}{\partial G} \times G^{\prime}(\gamma)
$$

we just show that the first term is negative. First notice that

$$
V=\frac{\sum_{i=1}^{n}\left(\frac{1}{G-\theta_{i}}\right) \pi_{i} \theta_{i}}{\sum_{i=1}^{n}\left(\frac{1}{G-\theta_{i}}\right) \pi_{i}}
$$

where $G=G(\gamma)$. Using the same steps as in the proof of Lemma 3.2 (c) in Veronesi (1998) we obtain that $\frac{\partial V}{\partial G}<0$ if and only if

$$
\sum_{i=1}^{n} \pi_{i}^{* *} \theta_{i}>\sum_{i=1}^{n} \pi_{i}^{*} \theta_{i}
$$

where

$$
\pi_{i}^{* *}=\frac{\frac{\pi_{i}}{\left(G-\theta_{i}\right)^{2}}}{\sum_{i=1}^{n} \frac{\pi_{i}}{\left(G-\theta_{i}\right)^{2}}}=\frac{\pi_{i}^{*}}{G-\theta_{i}}\left(\frac{\sum_{i=1}^{n} \frac{1}{G-\theta_{i}}}{\sum_{i=1}^{n} \frac{1}{\left(G-\theta_{i}\right)^{2}}}\right)
$$

and $\pi^{*}$ is defined in the proof of proposition 3. Since the last parenthesis is independent of $i$ and since $G-\theta_{i}$ is decreasing in $i$, we have that $\pi_{i}^{* *}>\pi_{i}^{*}$ for high indices $i$ and viceversa. Hence, the average computed using the distribution $\pi^{* *}$ must be higher than the average computed using the distribution $\pi^{*}$.

Finally, case (III) is immediate from direct computation: from the results in proposition 1 we can write:

$$
C_{2}=\frac{G(\gamma)-\theta_{1}+\lambda_{21}+\lambda_{12}}{D} ; \quad C_{1}=\frac{G(\gamma)-\theta_{2}+\lambda_{21}+\lambda_{12}}{D}
$$


where $D$ is just a positive common denominator. Hence, we can rewrite $A=C_{2}-C_{1}=\left(\theta_{2}-\theta_{1}\right) / D$ and the ratio: $B / A=\left(G(\gamma)-\theta_{2}+\lambda_{21}+\lambda_{12}\right) /\left(\theta_{2}-\theta_{1}\right)$ Since it is immediate to rewrite $\sigma_{S}=\iota_{x}\left(\Sigma \Sigma^{\prime}\right)^{\frac{1}{2}}+(\pi(1-$ $\pi) b) /(\pi+B / A)$, we see that $G^{\prime}(\gamma)>0$ implies that $\sigma_{S} \sigma_{S}^{\prime}$ decreases with $\gamma$.

\section{Appendix 2.}

Proof of Proposition 7 The Fourier Transform of the state-return density function over primitive states can be characterized by the usual Fokker-Planc backward equation (c.f., for example Karlin and Taylor 1982), which is the fundamental PDE above (30) along with the initial condition $f\left(z_{t}, \pi_{t}, 0\right)=\delta_{z-z_{t}}$, where $\delta$ stands for the Dirac function, and the last argument of $f(\cdot, \cdot)$ denotes time to maturity. Substituting $z=\log S$ into and letting $\tau \equiv T-t$ be the time to maturity implies that the fundamental PDE can be written as

$$
\begin{aligned}
r(\pi) f & =-\frac{\partial f}{\partial \tau}+\frac{\partial f}{\partial z}\left[r-\delta-\frac{1}{2}\left(\sigma_{x} \sigma_{x}^{\prime}+\frac{A^{2} \pi^{2}(1-\pi)^{2} b b^{\prime}}{(\pi A+B)^{2}}+2 \frac{A \pi(1-\pi)\left(\theta_{2}-\theta_{1}\right)}{\pi A+B}\right)\right] \\
& +\frac{\partial f}{\partial \pi}\left(\left(\lambda_{12}+\lambda_{21}\right)\left(\pi^{*}-\pi\right)-\pi(1-\pi) \gamma\left(\kappa_{2}-\kappa_{1}\right)\right)+\frac{1}{2} \frac{\partial^{2} f}{\partial z^{2}}\left(\sigma_{x} \sigma_{x}^{\prime}+\frac{A^{2} \pi^{2}(1-\pi)^{2} b b^{\prime}}{(\pi A+B)^{2}}+2 \frac{A \pi(1-\pi)\left(\theta_{2}-\theta_{1}\right)}{\pi A+B}\right) \\
& +\frac{\partial^{2} f}{\partial z \partial \pi}\left(\frac{\pi^{2}(1-\pi)^{2} A b b^{\prime}}{\pi A+B}+\pi(1-\pi) \sigma_{x} b^{\prime}\right)+\frac{1}{2} \frac{\partial^{2} f}{\partial \pi^{2}} \pi^{2}(1-\pi)^{2} b b^{\prime} .
\end{aligned}
$$

We recall that $r(\pi)=\phi+\gamma\left(\kappa_{2} \pi+\kappa_{1}(1-\pi)\right)-1 / 2 \gamma(\gamma+1) \sigma_{c} \sigma_{c}^{\prime}$ in equilibrium. Define

$$
\begin{aligned}
& A_{1}(\pi)=\left(\lambda_{12}+\lambda_{21}\right) \cdot\left(\pi^{*}-\pi\right)-\pi(1-\pi) \gamma\left(\kappa_{1}-\kappa_{2}\right), \\
& A_{2}(\pi)=\frac{A^{2} b b^{\prime}(1-\pi)^{2} \pi^{2}}{(\pi A+B)^{2}}+\sigma_{x} \sigma_{x}^{\prime}+\frac{2 A(1-\pi) \pi b \sigma_{x}^{\prime}}{\pi A+B}, \\
& A_{3}(\pi)=\frac{A b b^{\prime}(1-\pi)^{2} \pi^{2}}{\pi A+B}+(1-\pi) \pi\left(\theta_{1}-\theta_{2}\right), \quad \text { and }, \\
& A_{4}(\pi)=b b^{\prime} \pi^{2}(1-\pi)^{2} .
\end{aligned}
$$

Taking a Fourier Transform on both sides of the PDE (45) with respect to $z$ and using the frequency variable $\omega_{1}$ and $i=\sqrt{-1}$, implies

$$
\begin{aligned}
r(\pi) \bar{f} & =-\frac{\partial \bar{f}}{\partial \tau}-i \omega_{1} \bar{f}\left(r-\delta-\frac{1}{2} A_{2}(\pi)\right)+\frac{\partial \bar{f}}{\partial \pi} A_{1}(\pi) \\
& -\frac{\omega_{1}^{2}}{2} \bar{f} A_{2}(\pi)-i \omega_{1} \frac{\partial \bar{f}}{\partial \pi} A_{3}(\pi)+\frac{\partial^{2} \bar{f}}{\partial \pi^{2}} \frac{1}{2} A_{4}(\pi)
\end{aligned}
$$

with initial condition $\bar{f}\left(\omega_{1}, \pi, 0 ; z_{t}\right)=e^{i \omega_{1} z_{t}}$ (notice that the Fourier Transform depends on the initial $\log$ price, $z_{t}$ ). Now taking another Fourier Transform on both sides of the PDE (46) with respect to the time 
variable and using $\omega_{2}$ as the frequency variable implies that

$$
-e^{i \omega_{1} z_{t}}=\hat{f}\left(-r+i \omega_{2}-i \omega_{1}\left(r-\delta-\frac{1}{2} A_{2}(\pi)\right)-\frac{\omega_{1}^{2}}{2} A_{2}(\pi)\right)+\hat{f}_{\pi}\left(A_{1}(\pi)-i \omega_{1} A_{3}(\pi)\right)+\frac{1}{2} \hat{f}_{\pi \pi} A_{4}(\pi) .
$$

Now suppressing $\omega_{1}$ and $\omega_{2}$ for the time being, we further define

$$
\begin{aligned}
h_{1}(\pi) & =\frac{1}{2} A_{4}(\pi) \\
h_{2}(\pi) & =A_{1}(\pi)-i \omega_{1} A_{3}(\pi) \\
h_{3}(\pi) & =-r(\pi)+i \omega_{2}-i \omega_{1}\left(r(\pi)-\frac{1}{\pi A+B}\right)-\frac{\omega_{1}^{2}}{2} A_{2}(\pi)
\end{aligned}
$$

(47) can also be written concisely as $-e^{i \omega_{1} z_{t}}=h_{1}(\pi) \hat{f}_{\pi \pi}+h_{2}(\pi) \hat{f}_{\pi}+h_{3}(\pi) \hat{f}$. For given $\omega_{1}, \omega_{2},(47)$ is an ODE in $\pi$ with no known explicit analytical solution. Looking ahead, it is evident that the solution of the system of difference equations that will solve the ODE (provided at the end of this proof) is homogeneous of degree 1 in the forcing term $h_{0}$. Anticipating a future fourier inversion with respect to $\omega_{2}$, we first solve the slightly simpler equation $-1=h_{1}(\pi) \hat{f}_{\pi \pi}+h_{2}(\pi) \hat{f}_{\pi}+h_{3}(\pi) \hat{f}$. Due to homogeneity, the inverse fourier transform of the solution of the first equation with respect to $\omega_{2}$, will coincide with $-e^{i \omega_{1} z_{t}}$ times the fourier inversion with respect to $\omega_{2}$ of the solution of the latter equation (the logic is simply that $-e^{i \omega_{1} z_{t}}$ will pass out of the integral for the fourier inversion with respect to $\left.\omega_{2}\right)$.

We solve $-1=h_{1}(\pi) \hat{f}_{\pi \pi}+h_{2}(\pi) \hat{f}_{\pi}+h_{3}(\pi) \hat{f}$ with a series solution about an ordinary point of the ODE (c.f., for example, Rainville 1964, Boyce and DiPrima 1997). Because $A_{4}(0)=A_{4}(1)=0,0$ and 1 are singular points of the ODE. However, 0.5 is an ordinary point of the ODE, and the solution can be written in the form

$$
\hat{f}\left(\omega_{1}, \pi, \omega_{2}\right)=\sum_{n=0}^{\infty} a_{n} \cdot(\pi-.5)^{n}
$$

Define the functions

$$
\begin{aligned}
p(\pi) & \equiv \frac{\left(A_{1}(\pi)-i \omega_{1} A_{3}(\pi)\right)}{\frac{1}{2} A_{4}(\pi)}=\frac{h_{2}(\pi)}{h_{1}(\pi)} \\
q(\pi) & \equiv \frac{\left(-r+i \omega_{2}-i \omega_{1}(r-\delta)-\omega_{1}^{2} A_{2}(\pi)\right)}{\frac{1}{2} A_{4}(\pi)}=\frac{h_{3}(\pi)}{h_{1}(\pi)}
\end{aligned}
$$

By Theorem 5.3.1 in Boyce and DiPrima (1997), the radius of convergence of the series solutions is atleast as large as the minimum of the radii of convergence of the series for $p$ and $q \cdot{ }^{16}$ It is well known that, when $p$ and $q$ are ratios of complex polynomials (as is the case), then convergent series exist for these functions at $\pi_{0}$ when the denominator series of $p$ and $q$ does not vanish, i.e., $\frac{1}{2} A_{4}\left(\pi_{0}\right) \neq 0$. Further, the radius of convergence of $p$ and $q$ is atleast as large as the distance from $\pi_{0}$ to the nearest zero of the denominator

\footnotetext{
${ }^{16}$ This theorem has been attributed to Immanuel Lazarus Fuchs by several authors. See also Theorem 20 in Rainville (1964).
} 
series. Therefore, a series solution of the form in (48) converges atleast for all $\pi \in(0,1)$. The difference equation to be satisfied by the $a_{n}$, for $n=2, \cdots, \infty$, is provided below.

David (1997) (Property 1) showed that the updating process $\left\{\pi_{t}\right\}$ has 'entrance' boundaries at 0 and 1; neither boundary can be reached from the interior of the state space, but it is possible to consider processes that begin there. In such cases, it is impossible to impose arbitrary boundary conditions on the ODE (47) at 0 and 1 . The boundary conditions are determined implicitly by formulating the same ODE as $\pi \rightarrow 0,1$. Since $h_{1}(0)=h_{1}(1)=0$, to satisfy the entrance boundary conditions at 0 and 1 , the coefficients $a_{n}, n=1, \cdots, M$, must also satisfy the equations:

$$
\begin{aligned}
& h_{2}(0) \cdot \hat{f}_{\pi}(0)+h_{3}(0) \cdot \hat{f}(0)=-1, \\
& h_{2}(1) \cdot \hat{f}_{\pi}(1)+h_{3}(1) \cdot \hat{f}(1)=-1 .
\end{aligned}
$$

Because we approximate the series solution (48) by a finite expansion $\hat{f}^{M}=\sum_{n=0}^{M} a_{n}\left(\omega_{1}, \omega_{2}\right) \cdot(\pi-.5)^{n}$, substituting $\hat{f}^{M}(0)=\sum_{n=0}^{M} a_{n} \cdot(-.5)^{n}$, and $\hat{f}^{M}(1)=\sum_{n=0}^{M} a_{n} \cdot(.5)^{n}$ into (49) and (50) provide two further equations in $a_{n}, n=0, \cdots, M$. Therefore, overall there are $M$ equations in $m$ unknowns, and since none of the equations is redundant, they permit a unique solution of $\left(a_{0}, a_{1}, \cdots, a_{M}\right)$.

The coefficients $a_{n}, n=0, \cdots, M$, satisfying the difference equation shown below, are each polynomials in $\omega_{1}$ and $\omega_{2}$, and we can write these as functions $a_{n}\left(\omega_{1}, \omega_{2}\right)$. Performing a term-by-term numerical fourier inversion (the terms $(\pi-.5)^{n}$ pass out of the integration) of the solution (48) with respect to $\omega_{2}$ using the Fourier inversion formula provides

$$
b_{n}\left(\omega_{1}, T-t\right)=\frac{1}{\Pi} \int_{0}^{\infty} \operatorname{Re}\left[e^{-i \omega_{2}(T-t)} a_{n}\left(\omega_{1}, \omega_{2}\right)\right] d \omega_{2} .
$$

Overall, the M-term approximate solution to the Fourier Transform of the state return density function is given by ${ }^{17}$

$$
\bar{f}^{M}\left(\omega_{1}, \pi, T-t ; z_{t}\right)=e^{i \omega_{1} z_{t}} \sum_{n=0}^{M} b_{n}\left(\omega_{1}, T-t\right)(\pi-.5)^{n} .
$$

A second numerical fourier inversion, as shown below Proposition 7, then provide the values of the primitive securities used for pricing options. This is completely analogous to the solutions for option pricing with stochastic jumps and stochastic volatility such as in Heston (1993), Bates (1996), Bakshi et al. (1997), and Scott (1997).

\section{Coefficients for the Difference Equation}

We explicitly provide the difference equation that must be satisfied by the coefficients $a_{n}$, for $n=2, \cdots, M$. in the finite approximation of the series solution (48). We start by making the finite Taylor

\footnotetext{
${ }^{17}$ Writing the Fourier Transform in this form rather than performing a double fourier inversion on the solution (48) will be advantageous in the formulation of the 'delta' of the option price with respect to the stock price.
} 
Series expansions of the coefficient functions of the ODE (47). We define $\left(h_{i q}\right)$ for $q=1$ to $q=Q$ as the vector of coefficients of $h_{i} \cdot(\pi A+B)^{2}$ for $i=1,2,3$. It can be easily verified that $Q$ equals 7,6 , and 5 respectively for $h_{1}, h_{2}$, and $h_{3}$ respectively. Similarly $h_{0 q}, q=1,2,3$ are the coefficients of the expansion of $-(\pi A+B)^{2}$. To satisfy the ODE (47) standard series solutions methods (c.f., for example, Rainville 1964, Boyce and DiPrima 1997) imply that the coefficients must satisfy the following recursive system of equations.

$$
\begin{aligned}
& h_{01}=h_{11} \cdot 2 \cdot a_{2}+h_{21} \cdot a_{1}+h_{31} \cdot a_{0}, \\
& h_{02}=h_{11} \cdot 2 \cdot 3 \cdot a_{3}+h_{12} \cdot 2 \cdot a_{2}+h_{21} \cdot 2 \cdot a_{2}+h_{22} \cdot a_{1}+h_{31} \cdot a_{1}+h_{32} \cdot a_{0}, \\
& h_{03}=h_{11} \cdot 3 \cdot 4 \cdot a_{4}+h_{12} \cdot 2 \cdot 3 \cdot a_{3}+h_{13} \cdot 1 \cdot 2 \cdot a_{2}+h_{21} \cdot 3 \cdot a_{3}+h_{22} \cdot 2 \cdot a_{2}+h_{23} \cdot a_{1}+h_{31} \cdot a_{2} \\
& +h_{32} \cdot a_{1}+h_{33} \cdot a_{0}, \\
& 0=h_{11} \cdot 4 \cdot 5 \cdot a_{5}+h_{12} \cdot 4 \cdot 3 \cdot a_{4}+h_{13} \cdot 3 \cdot 2 \cdot a_{3}+h_{14} \cdot 2 \cdot a_{2}+h_{21} \cdot 4 \cdot a_{4}+h_{22} \cdot 3 \cdot a_{3} \\
& +h_{23} \cdot 2 \cdot a_{2}+h_{24} \cdot a_{1}+h_{31} \cdot a_{3}+h_{32} \cdot a_{2}+h_{33} \cdot a_{1}+h_{34} \cdot a_{0}, \\
& 0=h_{11} \cdot 5 \cdot 6 \cdot a_{6}+h_{12} \cdot 4 \cdot 5 \cdot a_{5}+h_{13} \cdot 3 \cdot 4 \cdot a_{4}+h_{14} \cdot 2 \cdot 3 \cdot a_{3}+h_{15} \cdot 2 \cdot a_{2}+h_{21} \cdot 5 \cdot a_{5} \\
& +h_{22} \cdot 4 \cdot a_{4}+h_{23} \cdot 3 \cdot a_{3}+h_{24} \cdot 2 \cdot a_{2}+h_{25} \cdot a_{1}+h_{31} \cdot a_{4}+h_{32} \cdot a_{3}+h_{33} \cdot a_{2}+h_{34} \cdot a_{1}+h_{35} \cdot a_{0} \text {, } \\
& 0=h_{11} \cdot 6 \cdot 7 \cdot a_{7}+h_{12} \cdot 5 \cdot 6 \cdot a_{6}+h_{13} \cdot 4 \cdot 5 \cdot a_{5}+h_{14} \cdot 3 \cdot 4 \cdot a_{4}+h_{15} \cdot 2 \cdot 3 \cdot a_{3}+h_{16} \cdot 1 \cdot 2 \cdot a_{2} \\
& +h_{21} \cdot 6 \cdot a_{6}+h_{22} \cdot 5 \cdot a_{5}+h_{23} \cdot 4 \cdot a_{4}+h_{24} \cdot 3 \cdot a_{3}+h_{25} \cdot 2 \cdot a_{2}+h_{26} \cdot a_{1}+h_{31} \cdot a_{5} \\
& +h_{32} \cdot a_{4}+h_{33} \cdot a_{3}+h_{34} \cdot a_{2}+h_{35} \cdot a_{1}, \\
& 0=h_{11} \cdot(m+2) \cdot(m+1) \cdot a_{m+2}+h_{12} \cdot(m+1) \cdot(m) \cdot a_{m+1}+h_{13} \cdot(m) \cdot(m-1) \cdot a_{m} \\
& +h_{14} \cdot(m-1) \cdot(m-2) \cdot a_{m-1}+h_{15} \cdot(m-2) \cdot(m-3) \cdot a_{m-2}+h_{16} \cdot(m-3) \cdot(m-4) \cdot a_{m-3} \\
& +h_{17} \cdot(m-4) \cdot(m-5) \cdot a_{m-4}+h_{21} \cdot(m+1) \cdot a_{m+1}+h_{22} \cdot(m) \cdot a_{m}+h_{23} \cdot(m-1) \cdot a_{m-1} \\
& +h_{24} \cdot(m-2) \cdot a_{m-2}+h_{25} \cdot(m-3) \cdot a_{m-3}+h_{26} \cdot(m-4) \cdot a_{m-4}+h_{31} \cdot a_{m}+h_{32} \cdot a_{m-1} \\
& +h_{33} \cdot a_{m-2}+h_{34} \cdot a_{m-3}+h_{35} \cdot a_{m-4}, \quad \text { for } m \geq 6 \text {. }
\end{aligned}
$$

To approximate the solution by a series of length $\mathrm{M}$, the set of M-2 equations are recursively solved for $a_{n}$, $n=2, \cdots, M$, as functions of $a_{0}$, and $a_{1}$. The boundary conditions provide the two remaining equations to solve the ODE.

Proof of Lemma 3. We closely follow the analysis in Chapter 15 of Karlin and Taylor (1982). In the two state case the posterior probability follows the diffusion process

$$
d \pi=\mu(\pi) d t+\sigma(\pi) d W
$$

The stationary density, $\Psi(x)$, of the diffusion process (52) must satisfy

$$
\frac{1}{2} \frac{\partial^{2}}{\partial y^{2}}\left[\sigma^{2}(y) \cdot \psi(y)\right]-\frac{\partial}{\partial y}[\mu(y) \cdot \psi(y)]=0,
$$


where under the risk-neutral measure $\mu(\pi)=\left(\lambda_{12}+\lambda_{21}\right)\left(\pi^{*}-\pi\right)-\gamma \cdot \pi \cdot\left(\kappa_{2}-\bar{\kappa}\right), \sigma(\pi)=\pi(1-\pi) b$, and $\pi^{*}=\frac{\lambda_{12}}{\lambda_{21}+\lambda_{21}}$ Solving the differential equation yields:

$$
\psi(x)=m(x)\left[C_{1} S(x)+C_{2}\right]
$$

where $s(x)=\exp \left(-2 \int^{x}\left[\mu[y] / \sigma^{2}(y)\right] d y\right), S(x)=\int^{x} s(x)$, and $m(x)=1 /\left[\sigma^{2}(x) s(x)\right] ; C_{1}$ and $C_{2}$ are constants that guarantee that $\psi(x) \geq 0$ on $(0,1)$ and $\int_{l}^{r} \psi(\xi) d \xi=1$. Because 0 and 1 are both entrance boundaries for $\pi_{t}, S(x) \rightarrow \infty$ as $x \rightarrow 0$ or $x \rightarrow 1$. In this case, $C_{2}$ is chosen equal to zero and the stationary density is

$$
\psi(x)=\frac{m(x)}{\int_{0}^{1} m(\xi) d \xi} .
$$

Now substituting $\mu(x)$, and $\sigma(x)$ into $m(x)$, completes the proof. The stationary density under the true measure is obtained by following the same steps, with $\mu(\pi)=\left(\lambda_{12}+\lambda_{21}\right)\left(\pi^{*}-\pi\right)$.

Proof of Corollary 7. Using the $M$ term polynomial approximation of the Fourier Transform $\bar{f}^{M}\left(\omega_{1}, \pi, T-\right.$ $\left.t ; z_{t}\right)$ in (6) and the characterization in Proposition 7, $B\left(\pi_{t}, T-t\right)=\sum_{n=0}^{M} b_{n}(0, T-t)(\pi-.5)^{n}$, and therefore $B_{\pi}\left(\pi_{t}, T-t\right)=\sum_{n=1}^{M} n b_{n}(0, T-t)(\pi-.5)^{n-1}$. Similarly, $G\left(\pi_{t}, T-t ; z_{t}\right)=e^{z_{t}} \sum_{n=0}^{M} b_{n}(1 / i, T-t)(\pi-.5)^{n}$, $G_{S}\left(\pi_{t}, T-t ; z_{t}\right)=G / S$, and $G_{\pi}\left(\pi_{t}, T-t ; z_{t}\right)=e^{z_{t}} \sum_{n=1}^{M} n b_{n}(1 / i, T-t)(\pi-.5)^{n-1}$. The functions $g_{1}$ and $g_{2}$ in (32) and (33) can be written as series whose partial derivatives are evident:

$$
\begin{aligned}
g_{1}\left(\omega_{1}, \pi, T-t ; z_{t}\right) & =\frac{e^{i \omega_{1} z_{t}} \sum_{n=0}^{M} b_{n}\left(1 / i+\omega_{1}, T-t\right)(\pi-.5)^{n}}{\sum_{n=0}^{M} b_{n}(1 / i, T-t)(\pi-.5)^{n}} \\
g_{2}\left(\omega_{1}, \pi, T-t ; z_{t}\right) & =\frac{e^{i \omega_{1} z_{t}} \sum_{n=0}^{M} b_{n}\left(\omega_{1}, T-t\right)(\pi-.5)^{n}}{\sum_{n=0}^{M} b_{n}(0, T-t)(\pi-.5)^{n}} .
\end{aligned}
$$

Now by passing partial derivatives under the integral of the fourier inversion, one obtains the desired functions; for example,

$$
\Pi_{j \pi}\left(z_{t}, K, \pi_{t}, T-t\right)=\frac{1}{\Pi} \int_{0}^{\infty} \operatorname{Re}\left[\frac{e^{-i \omega_{1} \log K} g_{j \pi}\left(\omega_{1}, \pi_{t}, T-t ; z_{t}\right)}{i \omega_{1}}\right] d \omega_{1} .
$$

Finally, using the product and chain rules of differentiation provide the stated expressions. 


\section{References}

Ait-Sahalia, Yacine, "Maximum-Likelihood Estimation of Discretely-Sampled Diffusions: A Closed-Form Approach," Technical Report, Princeton University 1998.

Akaike, Hirotugu, "Information Theory and the Extension of the Maximum Likelihood Principle," in Boris N. Petrov and Frigyes Csaki, eds., 2nd International Symposium on Information Theory, Budapest, Hungary: Akailseoniai-Kiudo, 1973.

Bakshi, Gurdip and Dilip Madan, "Spanning and Derivative Security Valuation," The Journal of Financial Economics (Forthcoming), 1999.

_ _ Charles Cao, and Zhiwu Chen, "Empirical Performance of Alternative Option Pricing Models," The Journal of Finance, 1997, 52 (5), 2003-2051.

_ _ _ a a _ _ _Do Call Prices and the Underlying Stock Always Move in the Same Direction," The Review of Financial Studies (Forthcoming), 1999.

Bates, David S., "Jumps and Stochastic Volatility: Exchange Rate Processes Implicit in PHLX Deutsche Mark Options," The Review of Financial Studies, 1996, 9 (1), 69-107.

— , "Post-'87 Crash Fears in the S\&P 500 Futures Option Market," Journal of Econometrics (Forthcoming), 1999.

Bergman, Yaacov, Bruce Grundy, and Zvi Weiner, "General Properties of Option Prices," The Journal of Finance, 1996, 51, 1573-1610.

Bollerslev, Timothy, R. Y. Chou, and K. F. Kroner, "ARCH Modelling in Finance. A Review of the Theory and Empirical Practice," Journal of Econometrics, 1992, 52, 5-59.

Boyce, William E. and Richard C. DiPrima, Elementary Differential Equations and Boundary Value Problems, John Wiley and Sons, New York, 1997.

Brennan, Michael J., "The Role of Learning in Dynamic Portfolio Decisions," European Finance Review, 1998.

Campbell, Sean D. and Canlin Li, "Option Prices with Unobserved and Regime-Switching Volatility," Technical Report, Department of Economics, University of Pennsylvania 1999. 
David, Alexander, "Fluctuating Confidence in Stock Markets: Implications for Returns and Volatility," Journal of Financial and Quantitative Analysis, 1997, 32 (4), 427-462.

Detemple, Jerome B., "Asset Pricing in a Production Economy with Incomplete Information," The Journal of Finance, 1986, 41, 383-391.

_ _ "Further Results on Asset Pricing with Incomplete Information," Journal of Economic Dynamics and Control, 1991, 15, 425-453.

Dickey, David A. and Wayne A. Fuller, "Distribution of the Estimators for Autoregressive Time Series with a Unit Root," Journal of the American Statistical Association, 1979, 74, $427-431$.

Dothan, Michael U. and David Feldman, "A Theory of Asset Prices and the Term Structure of Interest Rates in a Partially Observable Economy," The Journal of Finance, 1986, 41, 369-382.

Duffie, Darrell and David Lando, "The Term Structure of Credit Spreads with Incomplete Accounting Information," Mimeo, Finance Department, Stanford University 1999.

Dumas, Bernard, Jeff Fleming, and Robert Whaley, "Implied Volatility Functions: Empirical Tests," The Journal of Finance, 1998, 53 (6), 2059-2106.

Gennotte, Gerard, "Optimal Portfolio Choice Under Incomplete Information," The Journal of Finance, 1986, 41, 733-749.

Hamilton, James D., Time Series Analysis, Princeton University Press, Princeton, New Jersey, 1994.

Heston, Steven L., "A Closed-Form Solution for Options with Stochastic Volatility and Applications to Bond and Currency Options," The Review of Financial Studies, 1993, 6 (2), $326-343$.

Hull, John C., Options, Futures, and Other Derivative Securities, Prentice Hall, Englewood Cliffs, New Jersey, 1993.

and Alan White, "The Pricing of Options on Assets with Stochastic Volatility," Journal of Finance, 1987, 42 (2), 281-300.

Ingersoll, Jonathan E., Theory of Financial Decision Making, Rowman \& Littlefield, 1987. 
Jones, Robert A. and Joseph M. Ostroy, "Flexibility and Uncertainty," Review of Economic Studies, 1984, 51, 13-32.

Karlin, Samuel and Howard M. Taylor, A Second Course in Stochastic Processes, Academic Press, New York, 1982.

Kendall, Maurice and Alan Stuart, The Advanced Theory of Statistics, 4th edition, Volume 1, Macmillan Publishing Company, New York, 1977.

Liptser, Robert N. and A. N. Shiryaayev, Statistics of Random Processes I, Springer Verlag, New York, 1977.

Lo, Andrew W., "Maximum Likelihood Estimation of Generalized Ito Processes with DiscretelySampled Data," Econometric Theory, 1988, 4, 231-247.

Lucas, Robert E., "Asset Prices in an Exchange Economy," Econometrica, 1978, 46, 1429-1446.

Newey, Whitney R. and Kenneth D. West, "A Simple Positive Semi-Definite, Heteroscedasticity and Autocorrelation Consistent Covariance Matrix," Econometrica, 1987, 55, 703-708.

Rainville, Earl D., Intermediate Differential Equations, The Macmillan Company, New York, 1964.

Rubinstein, Mark, "Nonparametric Tests of Alternative Option Pricing Models Using All Reported Trades and Quotes on the 30 Most Active CBOE Option Classes from August 23, 1976 through August 31, 1978," Journal of Finance, 1985, 40, 455-480.

Scott, Louis O., "Pricing Stock Options in a Jump-Diffusion Model with Stochastic Volatility and Interest Rates: Applications of Fourier Inversion Methods," Mathematical Finance, 1997, $7(4), 413-426$.

Shimko, David, "Bounds of Probability," Risk, 1993, 6, 33-37.

Stein, Jeremy and E. M. Stein, "Stock Price Distributions with Stochastic Volatility," The Review of Financial Studies, 1991, 4, 727-752.

Veronesi, Pietro, "How Does Information Quality Affect Stock Returns?," The Journal of Finance (Forthcoming), 1999. 
__ , "Stock Market Overreaction to Bad News in Good Times: A Rational Expectations Equilibrium Model," The Review of Financial Studies (Forthcoming), 1999.

_ and Francis Yared, "Real and Nominal Bond Prices in a Regime-Shift Model," mimeo, University of Chicago - GSB 1999.

Whaley, Robert E., "Derivatives on Market Volatility: Hedging Tools Long Overdue," The Journal of Derivatives, 1993, 1 (1), 71-85.

Yan, Hong, "Uncertain Growth Prospects, Learning, and Asset Prices," Job Market Paper 1, University of California at Berkeley 1999.

Yared, Francis, "Path-Dependence in Expected Inflation: Evidence from a New Term-Structure Model," Ph.D. dissertation, University of Chicago - GSB 1999. 
Table 1: 2-State Model Calibrated to Real Earnings Growth on the S\&P 500: 1960-1998

\begin{tabular}{cccccc}
\hline$\theta_{1}$ & $\theta_{2}$ & $\lambda_{12}$ & $\lambda_{21}$ & $\sigma_{x}$ & $\rho_{x c}$ \\
\hline-.154 & .109 & .813 & .406 & .073 & .061 \\
\hline
\end{tabular}

Parameter estimates are based on maximum likelihood using the regime-switching estimation method as described in Hamilton (1994). 
Table 2: Implied Volatility Regression

Dependent Variable: Implied Volatility (Percent), $\mathcal{V}_{t}$

\begin{tabular}{|c|c|c|c|c|c|c|c|}
\hline No. & Constant & $\mathcal{V}_{t-1}$ & $\pi_{t}\left(1-\pi_{t}\right)$ & $\pi_{t}$ & $\pi_{t}^{2}$ & $\mathcal{V}_{t}^{U}$ & $\bar{R}^{2}$ \\
\hline 1. & $\begin{array}{l}17.03 \\
(1.01)^{*}\end{array}$ & & $\begin{array}{c}25.24 \\
(10.13)^{*}\end{array}$ & & & & 0.076 \\
\hline 2. & $\begin{array}{c}1.85 \\
(0.71)^{*}\end{array}$ & $\begin{array}{c}0.85 \\
(0.03)^{*}\end{array}$ & $\begin{array}{l}12.55 \\
(5.22)^{*}\end{array}$ & & & & 0.717 \\
\hline 3. & $\begin{array}{l}18.33 \\
(1.49)^{*}\end{array}$ & & & $\begin{array}{c}21.13 \\
(10.68)^{*}\end{array}$ & $\begin{array}{c}-22.70 \\
(10.44)^{*}\end{array}$ & & 0.074 \\
\hline 4. & $\begin{array}{l}2.49 \\
(.86)^{*}\end{array}$ & $\begin{array}{l}.84 \\
(.03)^{*}\end{array}$ & & $\begin{array}{c}10.64 \\
(4.80)^{*}\end{array}$ & $\begin{array}{r}-11.39 \\
(4.87)^{*}\end{array}$ & & 0.716 \\
\hline 5. & $\begin{array}{l}14.38 \\
(2.36)^{*}\end{array}$ & & & & & $\begin{array}{c}0.34 \\
(0.14)^{*}\end{array}$ & 0.079 \\
\hline 6. & $\begin{array}{l}-.25 \\
(1.41)\end{array}$ & $\begin{array}{c}.85 \\
(.03)^{*}\end{array}$ & & & & $\begin{array}{l}0.22 \\
(.09)^{*}\end{array}$ & 0.717 \\
\hline 7. & $\begin{array}{l}10.7 \\
(1.98)^{*}\end{array}$ & & & & & $\begin{array}{c}0.48 \\
(0.13)^{*}\end{array}$ & 0.168 \\
\hline
\end{tabular}

Standard errors are in parenthesis. The symbol * indicates statistical significance at the $5 \%$ level. The estimation uses the Newey and West (1987) consistent variance-covariance matrix. We reject the null hypothesis of a unit root for each of the series based on the Dickey and Fuller (1979) statistic. $\mathcal{V}_{t}$ is the CBOE Market Volatility Index, VIX. The belief process, $\pi_{t}$, is filtered from the calibrated model and is shown in Panel (B) of Figure 7. $\mathcal{V}_{t}^{U}$ is the ATM implied volatility for U option prices computed using Proposition 7 and the belief process. Lines 1 through 6 are based on a sample from 1986:1 through 1998:9, and Line 7 is based on the post-crash subsample from 1988:09 - 1989:09. 
Table 3: 3\% OTM Put-to-Call Price Regression (Skewness)

Dependent Variable: 3\% OTM Put-to-Call Price (Percent), $P_{t} / C_{t}$

\begin{tabular}{lccccl} 
No. & Constant & $P_{t-1} / C_{t-1}$ & $\pi_{t}$ & $P_{t}^{U} / C_{t}^{U}$ & $\bar{R}^{2}$ \\
\hline & & & & \\
1. & 119.05 & & 41.98 & & .062 \\
& $(7.16)^{*}$ & & $(15.21)^{*}$ & & .500 \\
2. & 36.19 & .68 & 15.07 & & .063 \\
& $(15.07)^{*}$ & $(.113)^{*}$ & $(7.75)^{*}$ & & .67 \\
3. & 73.37 & & & $(.22)^{*}$ & .503 \\
4. & $(22.26)^{*}$ & & & .69 & \\
& 18.50 & .25 & & $(.10)^{*}$ & \\
& $(16.02)$ & $(.11)^{*}$ & & &
\end{tabular}

Standard errors are in parenthesis. The symbol * indicates statistical significance at the $5 \%$ level. The estimation uses the Newey and West (1987) consistent variance-covariance matrix. We reject the null hypothesis of a unit root for each of the series based on the Dickey and Fuller (1979) statistic. Option prices and implied volatilities are from a sample of daily prices of S\&P 500 call and put options between 1986:04 and 1996:05 traded on the CBOE. The underlying index is obtained from the CBOE, and dividend yields are obtained from Standard and Poor's. The dependent variable is the monthly time-series of $3 \%$ OTM Put-to-Call Prices interpolated from available prices on the 15th (or closest to 15th) day of each month with approximately 30 days to maturity. The right-hand variable $P^{U} / C^{U}$ is computed from $\mathrm{U}$ option prices using Proposition 7 and the belief process filtered from the calibrated model shown in Panel (B) of Figure 7. 
Table 4: Convexity - Butterfly Spread Regression (Kurtosis)

$\underline{\text { Dependent Variable: Convexity Measure (Percent), } Y_{t}}$

No. Constant $\quad Y_{t-1} \quad \pi_{t}\left(1-\pi_{t}\right) \quad Y_{t}^{U} \quad \bar{R}^{2}$

\begin{tabular}{lccccc}
\hline & & & & \\
& & & & \\
& & & & \\
2.16 & & & \\
$2.54)^{*}$ & & $(4.41)^{*}$ & & .018 \\
& -.88 & .27 & -5.8 & & .081 \\
3. & $(.44)^{*}$ & $(.19)$ & $(2.70)^{*}$ & & .021 \\
& -.08 & & & 1.27 & \\
4. & $(.48)$ & & & $(.74)^{*}$ & .082 \\
& -.10 & .27 & & 1.01 & \\
& $(.45)$ & $(.19)$ & & $(.50)^{*}$ & \\
\hline
\end{tabular}

Standard errors are in parenthesis. The symbol * indicates statistical significance at the $10 \%$ level. The estimation uses the Newey and West (1987) consistent variance-covariance matrix. We reject the null hypothesis of a unit root for each of the series based on the Dickey and Fuller (1979) statistic. Option prices and implied volatilities are from a sample of daily prices of S\&P 500 call and put options between 1986:04 and 1996:05 traded on the CBOE. The underlying index is obtained from the CBOE, and dividend yields are obtained from Standard and Poor's. The dependent variable is a convexity measure defined as $Y_{t}=(\operatorname{Vol}(K / S=97)+\operatorname{Vol}(K / S=103)-2 \operatorname{Vol}(K / S=100))$, interpolated from available prices on the 15th (or closest to 15th) day of each month with approximately 30 days to maturity. $Y_{t}^{U}$ is the theoretical $Y_{t}$ using Proposition 7 at the fitted belief process $\pi_{t}$ shown in Panel (B) of Figure 7. 
Table 5: Hedging 'Violations'

Test Data (Number) Model (Number) Sample Size Data (Proportion) Model (Proportion)

Full Sample — Type I Errors:

$\begin{array}{llllll}\Delta C / \Delta S<0 & 147 & 163 & 1021 & .144 & .159 \\ \Delta P / \Delta S>0 & 191 & 150 & 1315 & .145 & .114\end{array}$

More Than 3 \% OTM Options - Type I Errors:

$\begin{array}{lrrrrr}\Delta C / \Delta S<0 & 54 & 121 & 330 & .164 & .367 \\ \Delta P / \Delta S>0 & 114 & 150 & 686 & .167 & .219\end{array}$

Full Sample — Type IV Errors:

\begin{tabular}{|c|c|c|c|c|c|}
\hline$\Delta C / \Delta S>1$ & 120 & 18 & 1021 & .117 & .017 \\
\hline$\Delta P / \Delta S<-1$ & 110 & 43 & 1315 & .084 & .032 \\
\hline
\end{tabular}

More Than 3 \% ITM Options - Type IV Errors:

\begin{tabular}{|c|c|c|c|c|c|}
\hline$\Delta C / \Delta S>1$ & 49 & 18 & 156 & .314 & .115 \\
\hline$\Delta P / \Delta S<-1$ & 27 & 25 & 100 & .250 & .270 \\
\hline
\end{tabular}

Data are daily changes in option prices between the 15th (the day closest to) and the 16th (the following trading day) of each month between 1986:4 and 1996:5. Option prices and implied volatilities are from a sample of daily prices of S\&P 500 call and put options between 1986:04 and 1996:05 traded on the CBOE. Model $\Delta^{U}$ are calculated using the deltas as shown in Corollary 7 for the belief process shown in Panel (B) of Figure 7 and the moneyness of each traded option. 
Figure 1: Examples of Historical Implied Volatility Curves

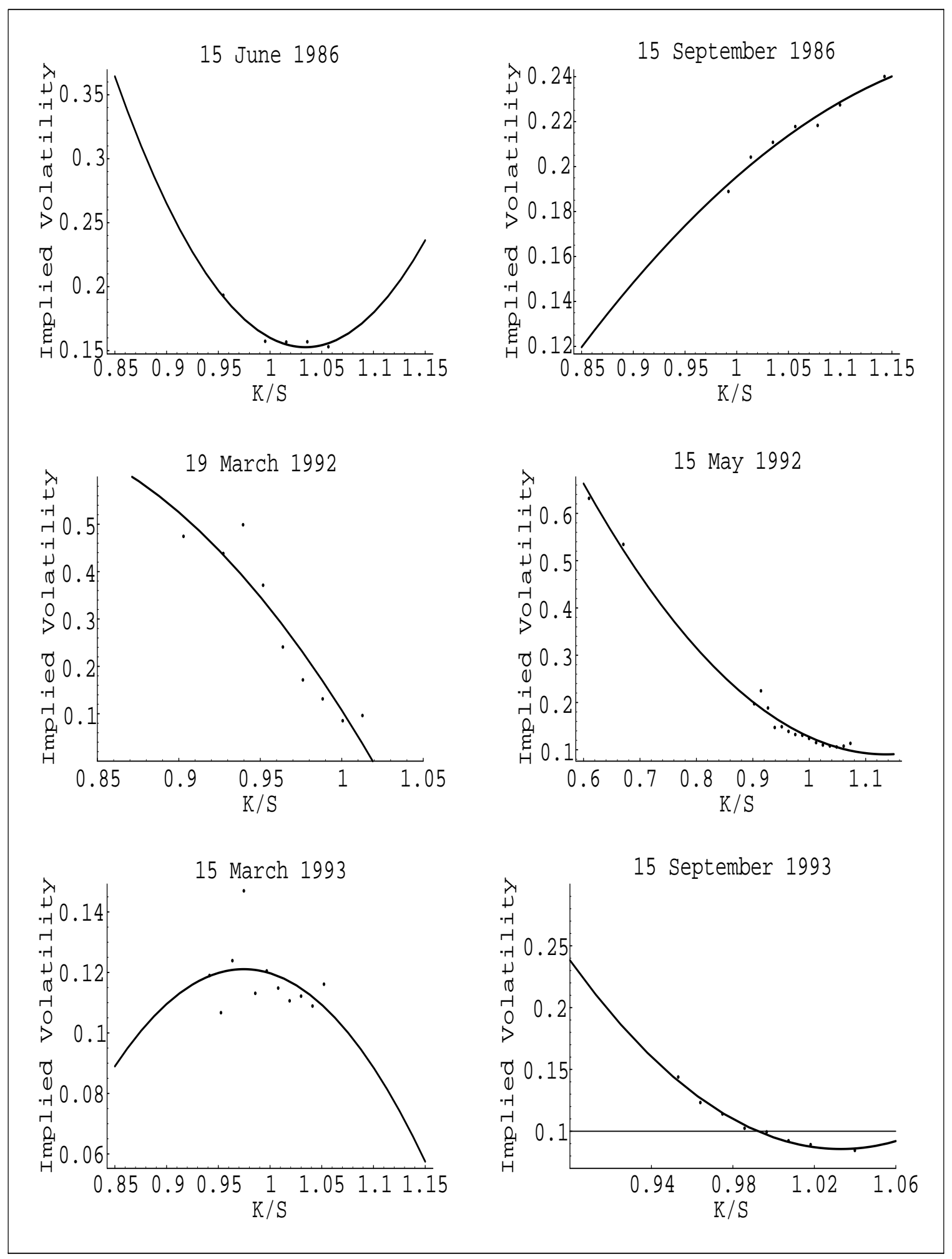

Implied volatility curves are displayed for the dates shown from a sample of daily prices of S\&P 500 call and put options between 1986:04 and 1996:05 traded on the Chicago Board Options Exchange. The underlying index is obtained from the Chicago Mercantile Exchange, and dividend yields are obtained from Standard and Poor's. A quadratic spline (polynomial of 3rd order) is fitted to all call options traded on the dates shown. The Akaike (1973) information criterion implies that 3rd order polynomials best fit the data at each of dates shown. 
Figure 2: Information Quality and Implied Volatility of U-Option Prices

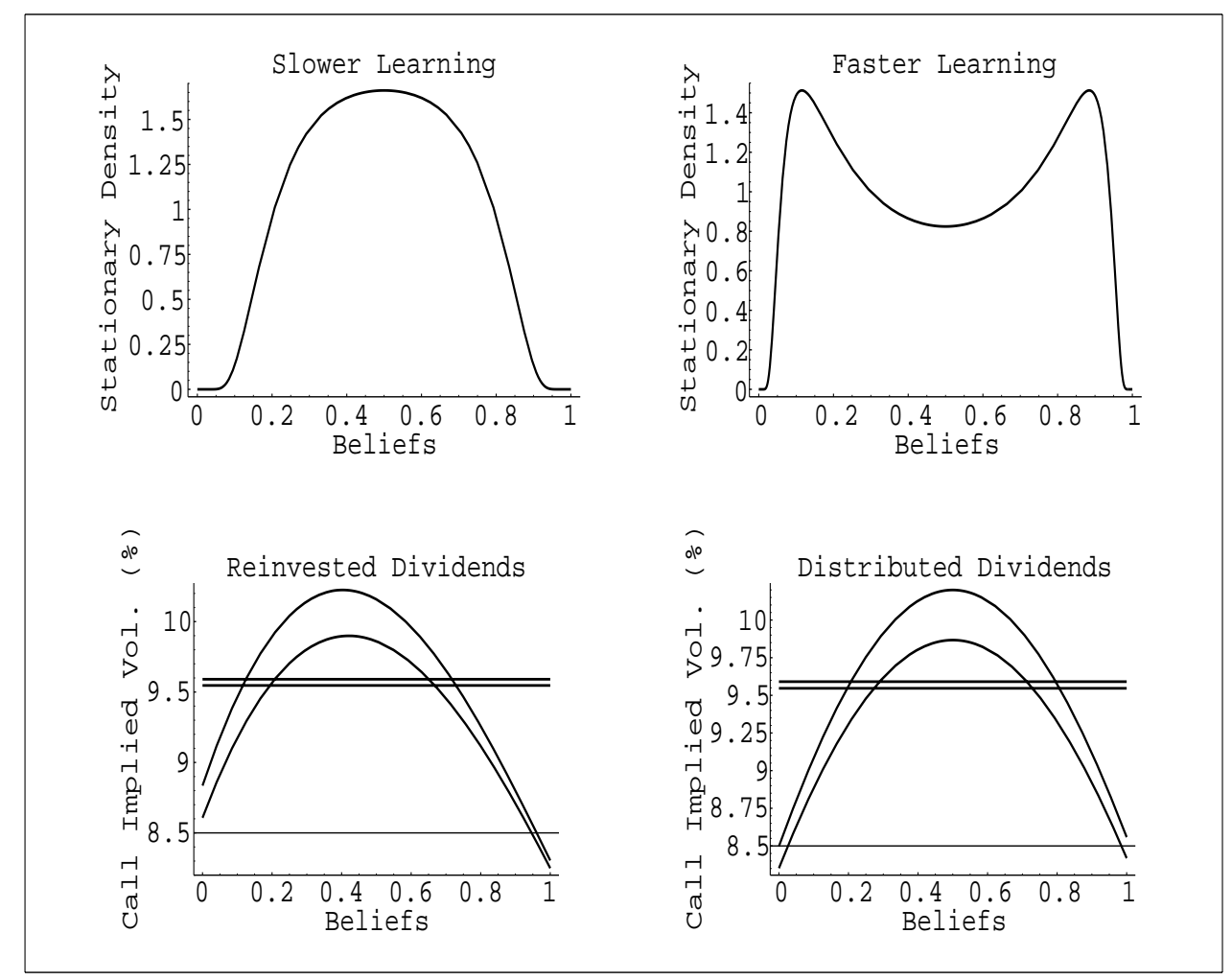

Top panel: Stationary density of the belief process $\pi$ under two different assumptions about the precision of signals about dividend growth. Left panel uses $\sigma_{2}=1000$ (uninformative signals), right panel uses $\sigma_{e}=.05$ (informative signal). The stationary densities in the top panels are computed using (34). Bottom panel: Implied volatility for a 6-month at-the-money call option obtained by equating the BS option price with the unconditional moments of dividends, with the $C^{\mathrm{U}}\left(z_{t}, K, \pi, T-t\right)$ price as given in (31). In each graph, the top curved line is for high precision of signals $\left(\sigma_{e}=.05\right)$. The straight horizontal line is the implied volatility for the unconditional stock volatility and dividend yield. The other parameters used are $\phi=.04$ (timediscount), $\gamma=2$ (risk-aversion), $\sigma_{c}=.036$ (consumption growth volatility), $\kappa_{1}=\kappa_{2}=\bar{\kappa}=.024$ (constant and observable growth rate of consumption), $\theta_{2}=.05$ (high growth rate of dividends), $\theta_{1}=-.05$ (low growth rate of dividends), $\lambda_{12}=.6, \lambda_{21}=.6$ (switching parameters), $\sigma_{x}=.073$ (volatility of earnings-growth) and $\rho_{x c}=.061$ (correlation between earnings and consumption growth). 
Figure 3: Asymmetry of Fundamentals' Growth Rates and Implied Volatility of U-Option Prices

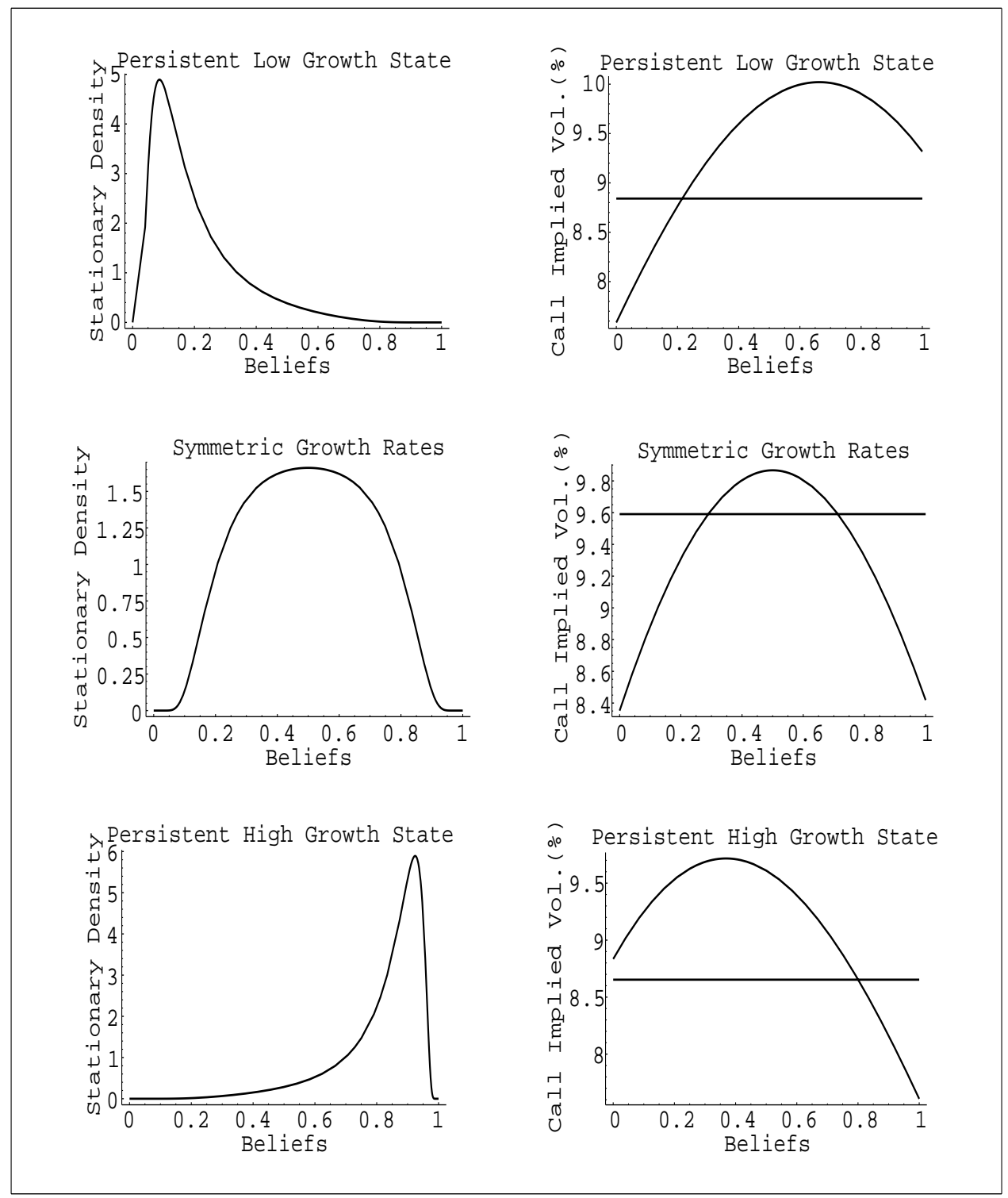

Left-hand panels plot stationary densities and right-hand panels plot the implied volatilities for 6-month, ATM call options obtained by equating the BS option price with the unconditional moments of dividends, with the $C^{\mathrm{U}}\left(z_{t}, K, \pi, T-t\right)$ price as given in (31). Straight lines are unconditional volatilities. Top panel: Persistent low growth rate with switching probabilities $\lambda_{21}=1, \lambda_{12}=.2$. Middle panel: Symmetric growth rates with $\lambda_{21}=.6, \lambda_{12}=.6$; Bottom panel: Persistent high growth rate with $\lambda_{21}=.2, \lambda_{12}=1$. The other parameters used are $\phi=.04$ (time-discount), $\gamma=2$ (risk-aversion), $\sigma_{c}=.036$ (consumption growth volatility), $\sigma_{x}=.073$ (earnings growth volatility), $\kappa_{1}=\kappa_{2}=\bar{\kappa}=.024$ (constant and observable growth rate of consumption), $\theta_{2}=.05$ (high growth rate of dividends), $\theta_{1}=-.05$ (low growth rate of dividends) $\sigma_{x}=.073$ (volatility of earnings-growth), $\rho_{x c}=.061$ (correlation between earnings and consumption growth) and $\sigma_{e}=1000$ (uninformative signal). The parameters imply that the average number of switches are the same for each set. The stationary densities in the left panels are computed using (34). The implied volatilities in the right panels are the volatilities that equate the BS option price with the unconditional moments of dividends, with the $C^{\mathrm{U}}\left(z_{t}, K, \pi, T-t\right)$ price as given in (31). 
Figure 4: Risk Aversion and Implied Volatility of U-Option Prices

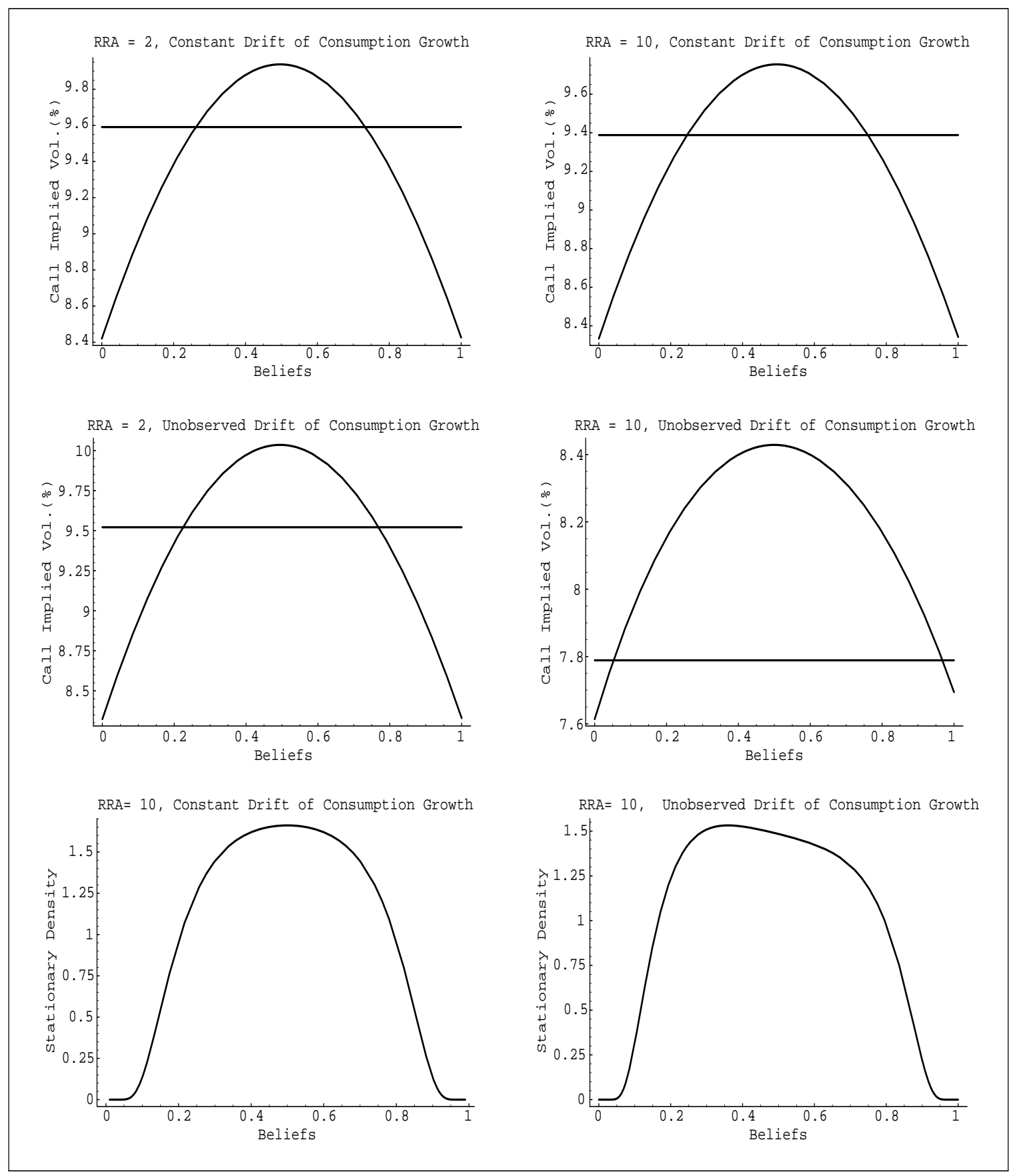

Implied volatilities are for 6 -month ATM call options obtained by equating the BS option price with the unconditional moments of dividends, with the $C^{\mathrm{U}}\left(z_{t}, K, \pi, T-t\right)$ price as given in (31). Straight lines are the unconditional volatilities. Top Panels: Observable and constant drift of consumption growth with $\kappa_{1}=\kappa_{2}=.024$; coefficient of risk aversion $\gamma=2$ for the left panel and $\gamma=10$ in the right panel. Middle Panels: Unobservable drift of consumption growth with $\kappa_{1}=.028$, and $\kappa_{2}=.02 ; \gamma=2$ for the left panel and $\gamma=10$ in the right panel. Bottom Panels: Stationary densities of the risk-neutral beliefs process given in Lemma 3 under observable and unobservable drifts respectively for $\gamma=10$. The parameters common to all panels are $\phi=.04$ (time-discount), $\sigma_{c}=.036$ (consumption growth volatility), $\theta_{1}=.05$ (high growth rate of dividends), $\theta_{2}=-.05$ (low growth rate of dividends), $\lambda_{12}=.5, \lambda_{21}=.5$ (switching parameters), $\sigma_{x}=.073$ (volatility of earnings-growth), $\rho_{x c}=.061$ (correlation between earnings and consumption growth) and $\sigma_{e}=1000$ (uninformative signal). 
Figure 5: Calibrated Model: 2-state case, 1960:01-1998:09

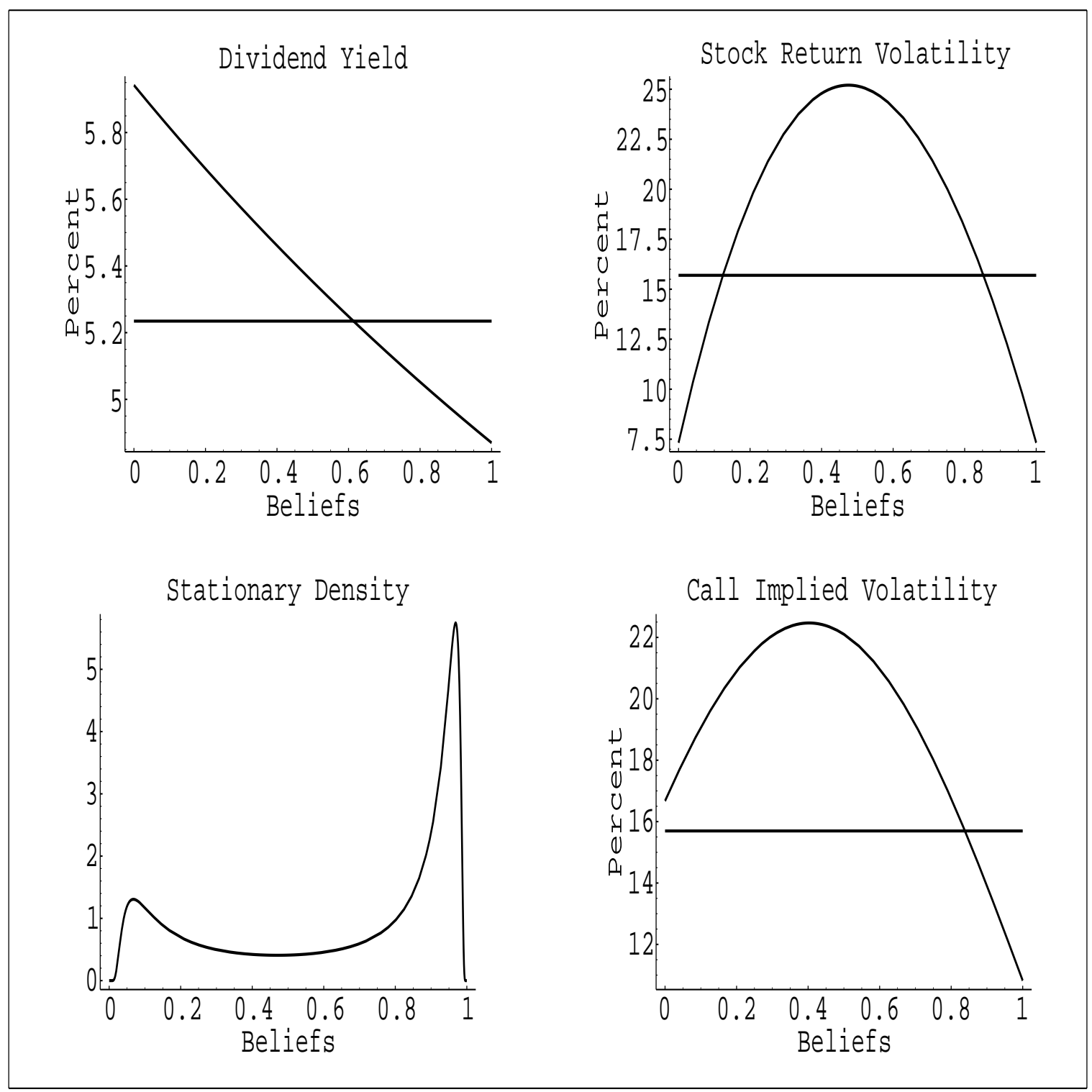

Top panels: The horizontal lines are the unconditional means of the variables shown computed by using the stationary density. Left panel plots the dividend yield, that equals $1 /(\pi A+B)$, where $A=C_{2}-C_{1}$, and $B=$ $C_{1}$, and the $C_{i}$ are computed using Proposition 1 . The right panel plots the volatility function $\sqrt{V}$, computed using (17) for the two-state case. Bottom panels: Left panel plots the stationary density computed using (34). The right panel plots the implied volatility for 6-months, at-the-money call options, obtained by equating the $\mathrm{BS}$ option price using the unconditional moments of dividends, with the $C^{\mathrm{U}}\left(x_{0}, K, \pi, T-t\right)$ price as given in (31). The other parameters used are those for the 2-state model calibrated to real earnings growth on the S\&P 500, as given in Table 1. The parameters are $\phi=.04$ (time-discount), $\gamma=2$ (risk-aversion), $\sigma_{c}=.036$ (consumption growth volatility), $\sigma_{x}=.073$ (earnings growth volatility), $\kappa_{1}=\kappa_{2}=\bar{\kappa}=.024$ (constant and observable growth rate of consumption), $\theta_{1}=.109$ (high growth rate of dividends), $\theta_{2}=-.154$ (low growth rate of dividends), $\lambda_{12}=.406, \lambda_{21}=.813$ (switching parameters), $\sigma_{x}=.073$ (volatility of earnings-growth), and $\rho_{x c}=.061$ (correlation between earnings and consumption growth). 
Figure 6: Smirks and Frowns in the Two-State Calibrated Model

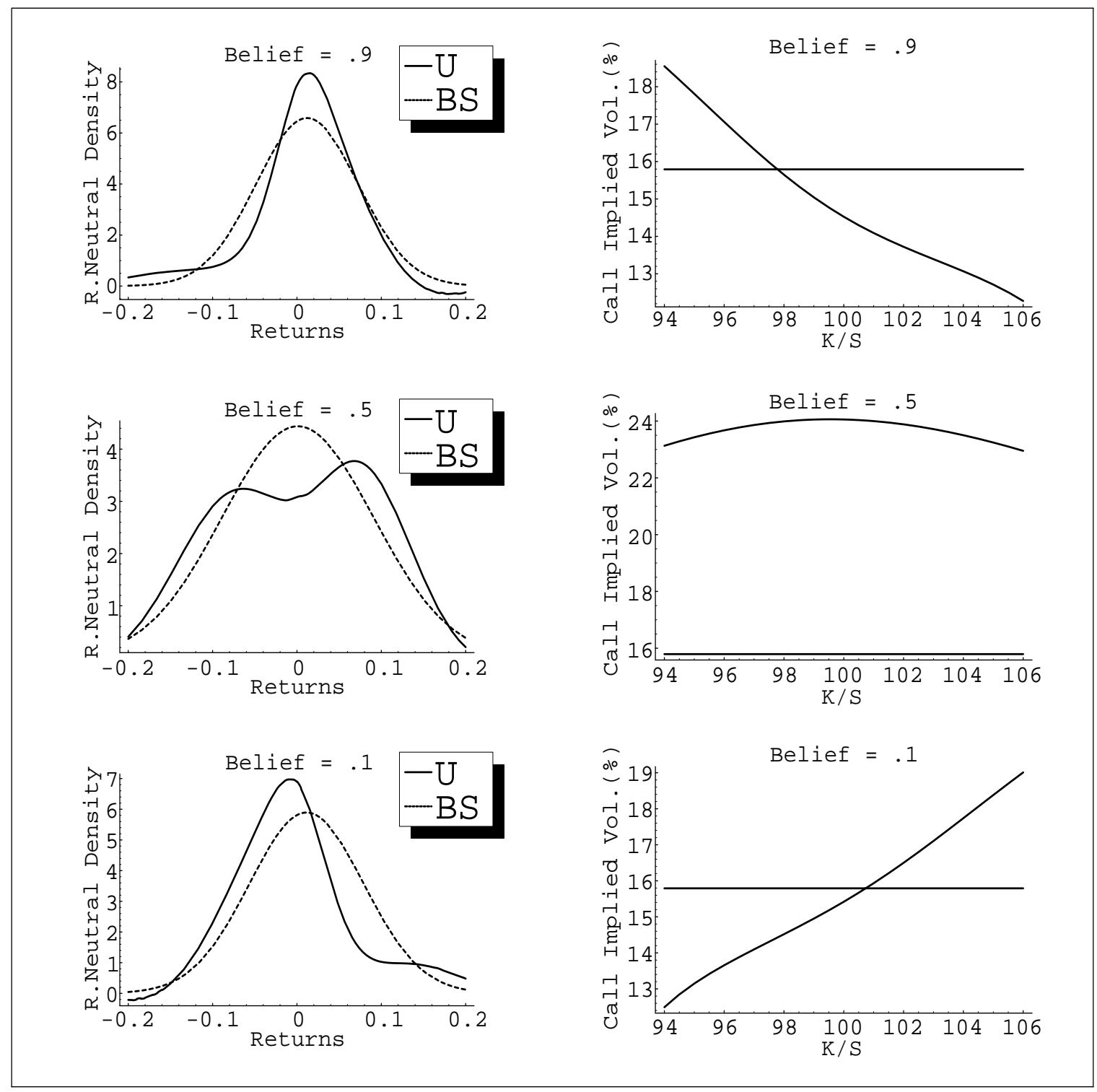

Left Panels: The state-return density functions conditional on three different initial beliefs $\pi=0.9$ (top panel), $\pi=0.5$ (center panel) and $\pi=0.1$ (bottom panel) with two months to maturity. The density is the inverse Fourier Transform of the state return density function given by $\bar{f}_{r}^{M}=\sum_{n=0}^{M} b_{n}\left(\omega_{1}, .167\right) \cdot(\pi-.5)^{n}$, and the $b_{n}$ are in (51) in Appendix 2. Right Panels: The corresponding implied volatility curves across different strike prices for call option prices with 2 months to maturity obtained by equating the BS option price with with the $C^{\mathrm{U}}\left(z_{t}, K, \pi, T-t\right)$ price as given in (31). The parameters used are those for the 2-state model calibrated to real earnings growth on the S\&P 500, as given in Table 1. The parameters are $\phi=.04$ (time-discount), $\gamma=2$ (risk-aversion), $\sigma_{c}=.036$ (consumption growth volatility), $\sigma_{x}=.073$ (earnings growth volatility), $\kappa_{1}=\kappa_{2}=\bar{\kappa}=.024$ (constant and observable growth rate of consumption), $\theta_{1}=.109$ (high growth rate of dividends), $\theta_{2}=-.154$ (low growth rate of dividends), $\lambda_{12}=.406, \lambda_{21}=.813$ (switching parameters), $\sigma_{x}=.073$ (volatility of earnings-growth), and $\rho_{x c}=.061$ (correlation between earnings and consumption growth). 
Figure 7: Two-State Filter on S\&P 500 Real Earnings Growth

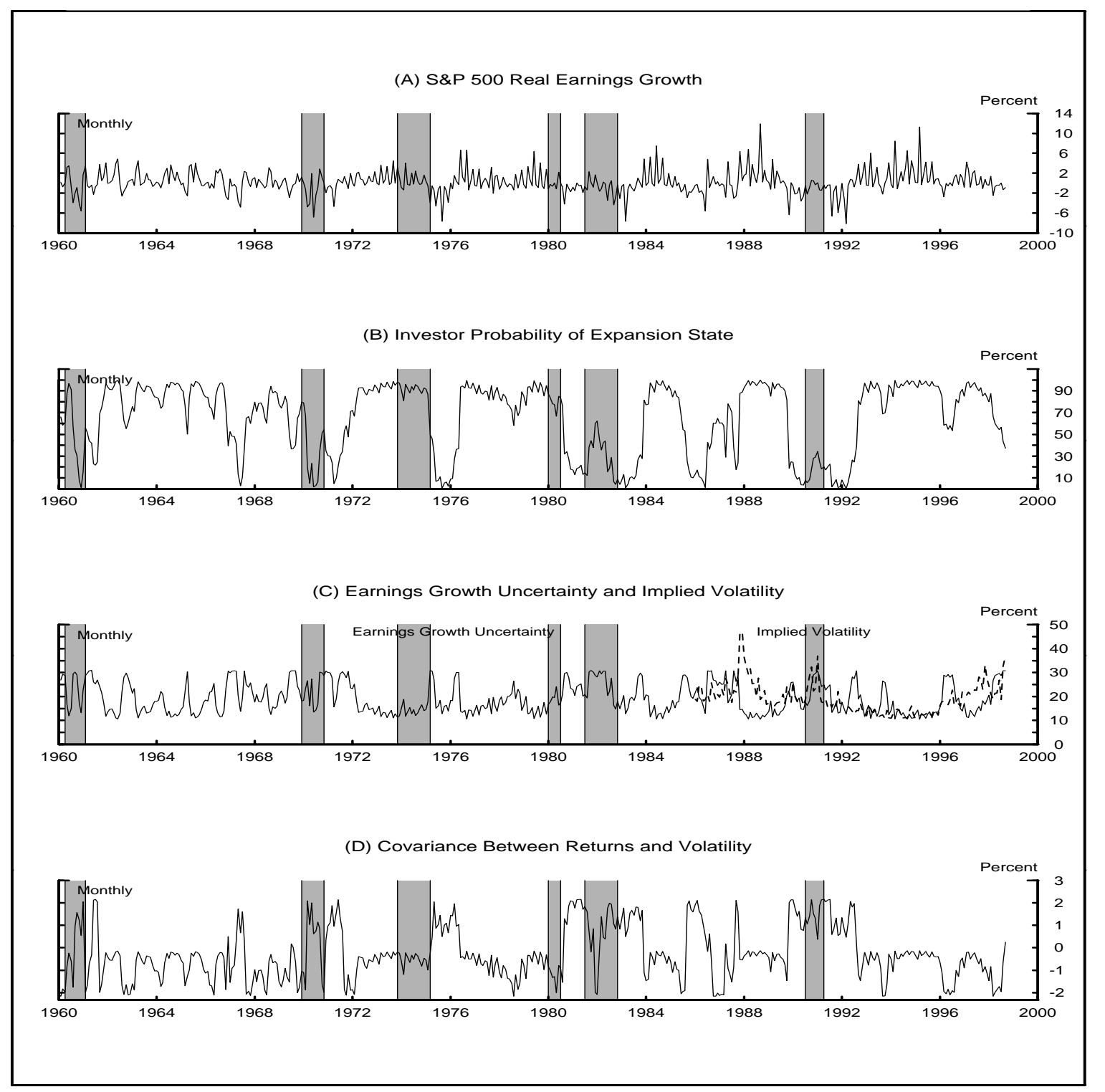

Panel (A) plots historical real earnings growth for S\&P 500 firms from 1960:01 to 1998:10. Panel (B) plots the filtered posterior probability to be in the high state. Panel (C) plots the ATM implied volatility for 1-month options that equates the $C^{\mathrm{U}}\left(z_{t}, K, \pi, T-t\right)$ price as given in (31) with the BS option price, using the belief process in (B) and the S\&P 100 implied volatility index (VIX) from 1986:1 to 1998:9. Panel (D) plots the covariance between returns and volatility $\operatorname{Cov}(d V, d R)=\sigma_{S} \sigma_{V}^{\prime}$ given by equation (23) in the text. 
Figure 8: Delta 'Violations'

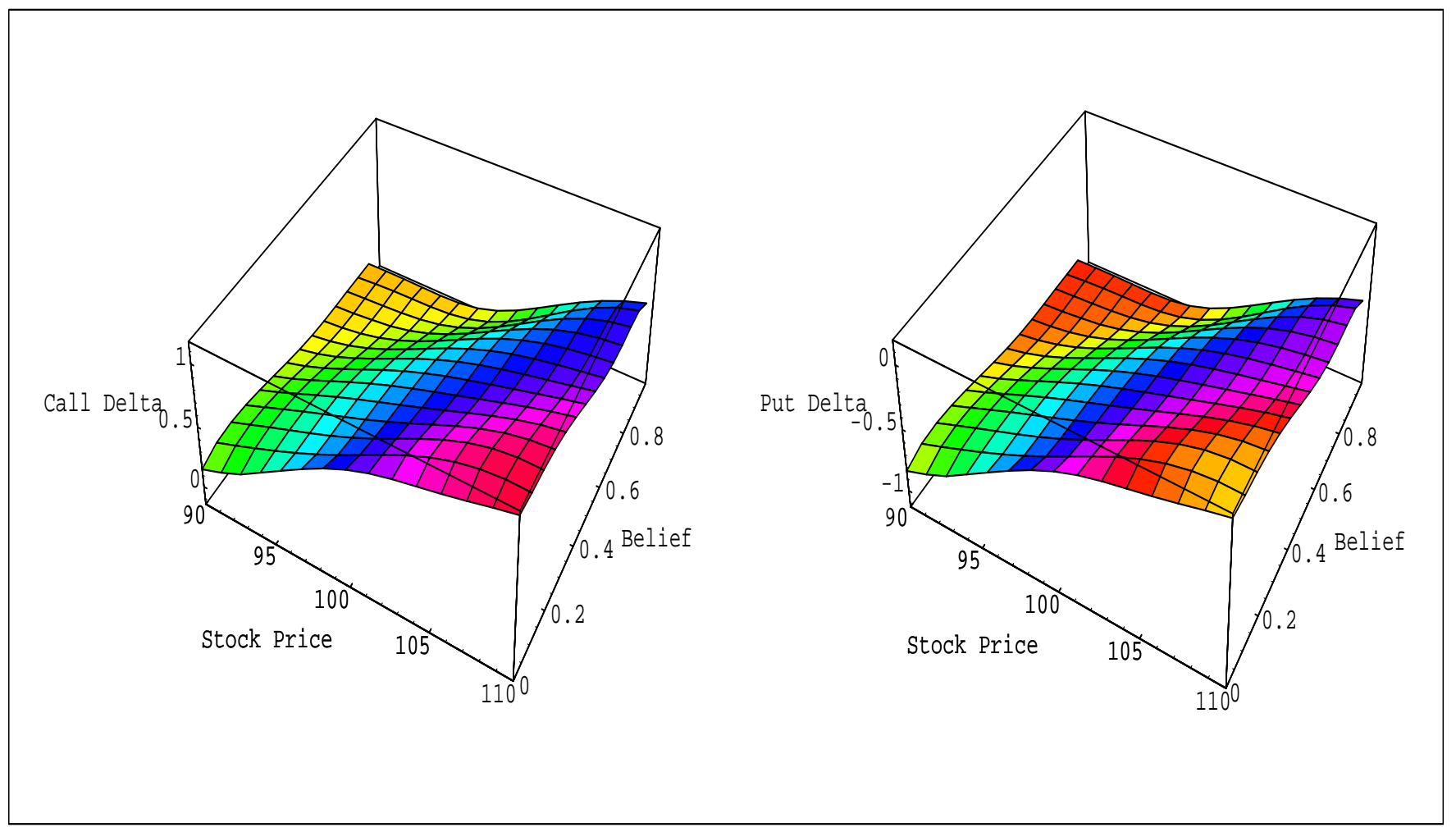

Observations satisfying $\Delta_{C}^{U}<0$, and $\Delta_{P}^{U}>0$ are classified as Type I violations, and $\Delta_{C}^{U}>1$, and $\Delta_{P}^{U}<-1$ as Type IV violations. Option deltas are calculated using Corollary 7 . The options have a strike price of $\$ 100$ and parameters are calibrated to the 2-state model on real earnings growth on the S\&P 500 between 1960:1 and 1998:9. The parameters are $\phi=.04$ (time-discount), $\gamma=2$ (risk-aversion), $\sigma_{c}=.036$ (consumption growth volatility), $\sigma_{x}=.073$ (earnings growth volatility), $\kappa_{1}=\kappa_{2}=\bar{\kappa}=.024$ (constant and observable growth rate of consumption), $\theta_{1}=.109$ (high growth rate of dividends), $\theta_{2}=-.154$ (low growth rate of dividends), $\lambda_{12}=.406, \lambda_{21}=.813$ (switching parameters), $\sigma_{x}=.073$ (volatility of earnings-growth), and $\rho_{x c}=.061$ (correlation between earnings and consumption growth). 